\title{
Adaptive Robust Backstepping Control of Permanent Magnet Synchronous Motor Chaotic System with Fully Unknown Parameters and External Disturbances
}

\author{
Yang Yu, Xudong Guo, and Zengqiang Mi \\ State Key Laboratory of Alternate Electrical Power System with Renewable Energy Sources, North China Electric Power University, \\ Baoding 071003, China
}

Correspondence should be addressed to Yang Yu; ncepu_yy@163.com

Received 15 December 2015; Revised 16 March 2016; Accepted 30 March 2016

Academic Editor: Shengjun Wen

Copyright (C) 2016 Yang Yu et al. This is an open access article distributed under the Creative Commons Attribution License, which permits unrestricted use, distribution, and reproduction in any medium, provided the original work is properly cited.

\begin{abstract}
The chaotic behavior of permanent magnet synchronous motor is directly related to the parameters of chaotic system. The parameters of permanent magnet synchronous motor chaotic system are frequently unknown. Hence, chaotic control of permanent magnet synchronous motor with unknown parameters is of great significance. In order to make the subject more general and feasible, an adaptive robust backstepping control algorithm is proposed to address the issues of fully unknown parameters estimation and external disturbances inhibition on the basis of associating backstepping control with adaptive control. Firstly, the mathematical model of permanent magnet synchronous motor chaotic system with fully unknown parameters is constructed, and the external disturbances are introduced into the model. Secondly, an adaptive robust backstepping control technology is employed to design controller. In contrast with traditional backstepping control, the proposed controller is more concise in structure and avoids many restricted problems. The stability of the control approach is proved by Lyapunov stability theory. Finally, the effectiveness and correctness of the presented algorithm are verified through multiple simulation experiments, and the results show that the proposed scheme enables making permanent magnet synchronous motor operate away from chaotic state rapidly and ensures the tracking errors to converge to a small neighborhood within the origin rapidly under the full parameters uncertainties and external disturbances.
\end{abstract}

\section{Introduction}

In recent years, the permanent magnet synchronous motor (PMSM) is utilized widely in various industrial fields due to its constantly dropping production cost, simple structure, high torque, and high efficiency. However, Hemati found that PMSM would generate chaotic behavior with system parameters entering into a certain region [1]. Previous studies have shown that the chaotic movement of PMSM will produce irregular oscillations of torque and speed, exacerbate current noise, and worsen operation performance and may even damage the entire drive system. Therefore, research on PMSM chaos phenomenon has attracted extensive attention worldwide [2-5], and further studying on the control method of PMSM chaos is of extreme significance [6-8].

The nonlinear characteristics of PMSM, such as multivariability, strong coupling, and high dimension, make it difficult to control for traditional linear control theory. Hence, a variety of modern and nonlinear control algorithms are introduced to suppress PMSM chaotic behavior. In terms of these control algorithms whether or not relying on the model parameters, the previous control methods can primarily be divided into two categories. The first type is on the basis of accurate model parameters, such as entrainment and migration control [9], exact feedback linearization control [10], and decoupling control [11]. However, the accuracy of these control methods directly depends on PMSM model parameters; if the system parameters deviate from the rated values, the control performance will go bad. The second type is based on unknown parameters, which have become the research focus of PMSM chaos suppression recently, mainly including sliding mode variable structure control [12, 13], fuzzy control [14], and $H_{\infty}$ control [15]. However, sliding mode variable structure control requires uncertain parameters to satisfy certain matching conditions, fuzzy control is 
dependent on the fuzzification of Takagi-Sugeno, and $H_{\infty}$ control is inclined to ignore the operating states under special conditions [16]. In essence, PMSM chaotic system is highly sensitive to initial states and parameters, and PMSM model parameters are susceptible to the temperature and humidity of the surrounding environment. Therefore, PMSM chaotic repression with unknown model parameters has applicability to a broader field and is more in line with reality [17]. Actually, the adaptive control (AC) provides a natural routine for PMSM chaotic control with unknown parameters, which has been presented in literatures $[12,13,18]$.

Backstepping control (BC) is one of the most popular nonlinear control methods newly proposed to address parameter uncertainty, specifically the uncertainty not satisfying matching condition, which has been successfully applied to many engineering fields such as motor drive, temperature control of boiler main steam, and rocket location tracking. The core idea of $\mathrm{BC}$ is that complex highdimensional nonlinear systems are decomposed into many simple low-dimensional subsystems and virtual control variables are introduced to backstepping process to design concrete controllers. In addition, BC has been successfully applied to suppress Liu chaotic system [19] and Chen chaotic system [20]. Therefore, the idea of combining BC with AC provides a useful and feasible train of thought to control PMSM chaotic system with unknown parameters. Literatures $[21,22]$ have exactly practiced this idea.

However, the conventional backstepping approach is confronted with two major problems of solving complicated "regression matrix" [23] and encountering "explosion of terms" [24]. In [25], the complexity of regression matrix is sufficiently manifested, which almost occupies one full page. Nevertheless, explosion of terms is an inherent shortcoming and is induced by repeated differentiations of virtual variables, particularly in design of adaptive backstepping controller [26]. Additionally, integration of BC with AC is frequently faced with the singularity arising from any estimation term emerging as a denominator of any control input. The overparameterization caused by the number of estimations larger than actual system parameters hinders the conventional adaptive backstepping control.

In addition to the above problems, to the extent of our knowledge, mostly existing literatures on PMSM chaotic control only concentrate on the cases of single unknown parameter and partial unknown parameters [21, 22], and there is no way to address the issue of fully unknown parameters. Furthermore, the existing researches mainly aim at the situation of sudden power failure during PMSM operation [16]; the existing conclusions lack the generality. Hence, through combination of $\mathrm{BC}$ and $\mathrm{AC}$, not only does this paper study the control issue of PMSM chaos suppression with fully unknown parameters, but also the external disturbances are taken into account in PMSM chaos model. Newly adaptive updating laws of unknown parameters are designed to totally estimate unknown parameters of PMSM chaotic model, and adaptive robust backstepping controllers on the basis of adaptive estimations and external disturbances are developed to drive PMSM to escape out of chaotic state quickly, inhibit the external disturbances, and accomplish the given signals tracking rapidly. The method proposed in this paper expands the applied range of backstepping control theory in PMSM chaotic system. Moreover, the study of chaos control problem with totally unknown parameters and external disturbances is more general and practical, and the results and conclusions obtained are more applicable.

\section{PMSM Chaotic Model with Fully Unknown Parameters}

For a PMSM, its mathematical model in $d q$ axis coordinate system can be described as follows [16]:

$$
\begin{aligned}
& \dot{\bar{\omega}}=\frac{3 p \phi_{m}}{2 J} \bar{i}_{q}-\frac{B}{J} \bar{\omega}-\frac{\bar{\tau}_{l}}{J}, \\
& \dot{\bar{i}}_{q}=-\frac{R}{L_{q}} \bar{i}_{q}-p \bar{\omega} \cdot \bar{i}_{d}-\frac{p \phi_{m}}{L_{q}} \cdot \bar{\omega}+\frac{1}{L_{q}} \bar{u}_{q}, \\
& \dot{\bar{i}}_{d}=-\frac{R}{L_{d}} \bar{i}_{d}+p \bar{\omega} \cdot \bar{i}_{q}+\frac{1}{L_{d}} \bar{u}_{d},
\end{aligned}
$$

where $\bar{\omega}$ is the mechanical angular velocity of the rotating rotor, $\hat{i}_{d}$ and $\hat{i}_{q}$ are $d$ axis and $q$ axis currents of stator winding, respectively, $\bar{u}_{d}$ and $\bar{u}_{q}$ are $d$ axis and $q$ axis voltages of stator winding, $p$ is the number of rotor pole pairs, $\phi_{m}$ is the flux generated by permanent magnets, $J$ is the moment of inertia, $B$ is the viscous damping coefficient, $\widehat{\tau}_{l}$ is the load torque, $R$ is the phase resistance of the stator windings, and $L_{d}$ and $L_{q}$ are $d$ axis and $q$ axis inductances of stator winding, respectively. For a PMSM with uniform air gap, $L_{d}=L_{q}$. Hence, we use $L$ to substitute $L_{d}$ and $L_{q}$ in the following paper.

Selecting the affine transformation $\left[\begin{array}{c}\bar{\omega} \\ \bar{i}_{q} \\ \bar{i}_{d}\end{array}\right]=$ $\left[\begin{array}{ccc}1 / p \tau & 0 & 0 \\ 0 & k & 0 \\ 0 & 0 & k\end{array}\right]\left[\begin{array}{l}\omega \\ i_{q} \\ i_{d}\end{array}\right]$ and time scale transformation $\bar{t}=\tau t$, the PMSM mathematical model described in (1) can be converted into dimensionless form as follows:

$$
\begin{aligned}
& \dot{\omega}=\frac{1}{\delta}\left(i_{q}-\omega\right)-\tau_{l}, \\
& \dot{i}_{q}=-i_{q}-\omega \cdot i_{d}+\gamma \cdot \omega+u_{q}, \\
& \dot{i}_{d}=-i_{d}+\omega \cdot i_{q}+u_{d},
\end{aligned}
$$

where $\tau=L / R, k=2 B / 3 p^{2} \tau \phi_{m}, u_{d}=(1 / k R) \bar{u}_{d}, \gamma=-\phi_{m} / k L$, $u_{q}=(1 / k R) \widehat{u}_{q}, \delta=J / B \tau$, and $\tau_{l}=p \tau^{2} \bar{\tau}_{l} / J$.

As presented in (2), the dynamic performance of PMSM depends on three parameters $\delta, \gamma$, and $\tau_{l}$. Considering the most general case, let $\delta=0.2, \gamma=50, \tau_{l}=3.2, u_{d}=-0.6$, and $u_{q}=0.8$. If the initial state is selected as $\left(\omega, i_{q}, i_{d}\right)=$ $(0,0,0)$, PMSM system will run on a chaotic state and display the chaotic behavior. A typical chaotic attractor of PMSM is manifested in Figure 1.

In reality, the three parameters $\delta, \gamma$, and $\tau_{l}$ in (2) tend to be unknown or to have uncertainties resulting from operating conditions. In other words, when all the parameters $\delta, \gamma$, and $\tau_{l}$ cannot be determined, (2) actually represents PMSM chaotic system model with fully unknown parameters. 


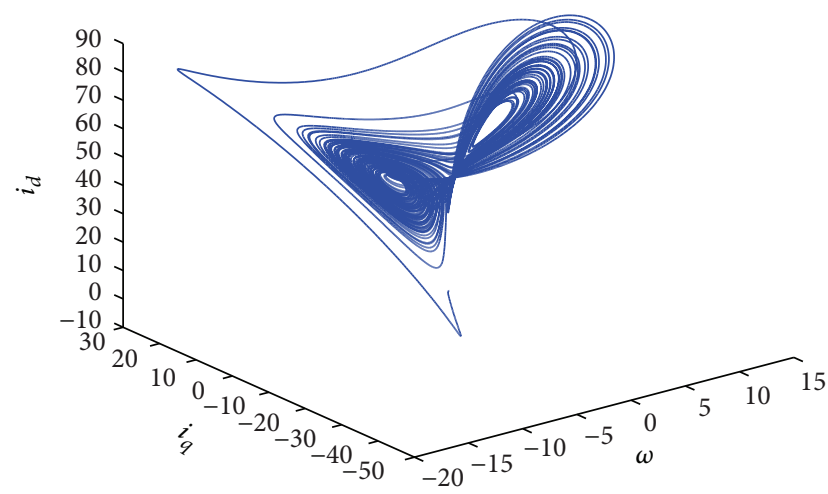

FIgURE 1: Chaotic attractor of PMSM system.

\section{Design of Adaptive Robust Controller with Backstepping Approach}

Taking a more general situation into account, the PMSM chaotic model described in (2) is immersed by external disturbances. The model can be rewritten as follows:

$$
\begin{aligned}
& \dot{\omega}=\frac{1}{\delta}\left(i_{q}-\omega\right)-\tau_{l}, \\
& \dot{i}_{q}=-i_{q}-\omega \cdot i_{d}+\gamma \cdot \omega+u_{q}+\Delta_{1}(\mathbf{x}, t), \\
& \dot{i}_{d}=-i_{d}+\omega \cdot i_{q}+u_{d}+\Delta_{2}(\mathbf{x}, t),
\end{aligned}
$$

where $\Delta_{1}(\mathbf{x}, t)$ and $\Delta_{2}(\mathbf{x}, t)$ represent the external disturbances, $\mathbf{x}$ indicates the system states, and $\mathbf{x}=\left(x_{1}, x_{2}, x_{3}\right)=$ $\left(\omega, i_{q}, i_{d}\right)$.

3.1. Control Objective and Assumptions. Control problem in the paper can be described as follows: for PMSM chaotic system (3) with fully unknown parameters $\delta, \gamma$, and $\tau_{l}$ and external disturbances $\Delta_{1}$ and $\Delta_{2}$, adaptive laws of unknown parameters $\delta, \gamma$, and $\tau_{l}$ are designed and adaptive robust controllers $u_{d}$ and $u_{q}$ are constructed to ensure PMSM breaks away from chaos rapidly and runs into an expected orbit. Simultaneously, the fully unknown parameters $\delta, \gamma$, and $\tau_{l}$ can be estimated accurately and the external disturbances can be inhibited effectively.

For convenience of controller design, the control system is supposed to hold some reasonable assumptions as follows.

Assumption 1. The state variables for PMSM chaotic system $\left(\omega, i_{q}, i_{d}\right)$ are observable.

Assumption 2. The external disturbances $\Delta_{i}(\mathbf{x}, t)$ satisfy the condition $\left|\Delta_{i}(\mathbf{x}, t)\right| \leq d_{i}(\mathbf{x}) f_{i}(t), i=1,2$, where $d_{i}(\mathbf{x})$ is a known function, $f_{i}(t)$ is an unknown but bounded timevarying function, and $\left|f_{i}(t)\right| \leq f_{\text {imax }}$, where $f_{i \max }$ is a constant.

Assumption 3. The desired speed and $d$ axis current reference signals $\omega^{*}$ and $i_{d}^{*}$ and their derivatives are known and bounded.
The estimated values of unknown system parameters are described as $\widehat{\delta}, \widehat{\gamma}$, and $\widehat{\tau}_{l}$; then, the estimation errors $\widetilde{\delta}, \widetilde{\gamma}$, and $\tilde{\tau}_{l}$ can be expressed as follows:

$$
\begin{aligned}
\widetilde{\delta} & =\widehat{\delta}-\delta, \\
\widetilde{\gamma} & =\widehat{\gamma}-\gamma, \\
\tilde{\tau}_{l} & =\widehat{\tau}_{l}-\tau_{l} .
\end{aligned}
$$

3.2. Controller Design. The essence of adaptive robust backstepping controller is to design controller through combination of backstepping method and adaptive approach; then, a reasonably stable function is built in accordance with Lyapunov stability theory to guarantee error variables to be effectively stabilized and meanwhile ensure the output of closed loop system tracks reference signals quickly. On the basis of this, the adaptive robust backstepping controller is designed as follows.

Step 1. For the speed reference signal $\omega^{*}$, define the tracking error $e_{\omega}$ as follows:

$$
e_{\omega}=\omega-\omega^{*} \text {. }
$$

Taking PMSM chaotic system model (3) into account, the derivative of (5) can be written as

$$
\dot{e}_{\omega}=\dot{\omega}-\dot{\omega}^{*}=\frac{1}{\delta}\left(i_{q}-\omega\right)-\tau_{l}-\dot{\omega}^{*} .
$$
follows:

Define the tracking error $e_{q}$ of $q$ axis stator current $i_{q}$ as

$$
e_{q}=i_{q}-i_{q}^{*}
$$

where $i_{q}^{*}$ is the expected output value of $i_{q}$.

For the $d$ axis current reference signal $i_{d}^{*}$, its tracking error $e_{d}$ is defined as follows:

$$
e_{d}=i_{d}-i_{d}^{*}
$$

By substitution of (7) into (6), we can obtain

$$
\begin{aligned}
\dot{e}_{\omega} & =\frac{1}{\delta}\left(e_{q}+i_{q}^{*}-\omega\right)-\tau_{l}-\dot{\omega}^{*} \\
& =\frac{1}{\delta}\left(i_{q}^{*}-\omega\right)-\widehat{\tau}_{l}+\widetilde{\tau}_{l}-\dot{\omega}^{*} .
\end{aligned}
$$

Let

$$
\begin{aligned}
& i_{q}^{*}=\omega+\widehat{\delta}\left(\widehat{\tau}_{l}+\dot{\omega}^{*}-k_{1} e_{\omega}\right), \\
& i_{d}^{*}=0
\end{aligned}
$$

where $k_{1}$ represents the positive control gain.

Through substitution of (10) into (9), (12) can be obtained:

$$
\begin{aligned}
\dot{e}_{\omega} & =\frac{1}{\delta} e_{q}+\frac{\widehat{\delta}}{\delta}\left(\widehat{\tau}_{l}+\dot{\omega}^{*}-k_{1} e_{\omega}\right)-\widehat{\tau}_{l}+\widetilde{\tau}_{l}-\dot{\omega}^{*} \\
& =\frac{1}{\delta} e_{q}+\frac{\delta+\widetilde{\delta}}{\delta}\left(\widehat{\tau}_{l}+\dot{\omega}^{*}-k_{1} e_{\omega}\right)-\widehat{\tau}_{l}+\widetilde{\tau}_{l}-\dot{\omega}^{*} \\
& =\frac{1}{\delta} e_{q}+\frac{\widehat{\delta}}{\delta}\left(\widehat{\tau}_{l}+\dot{\omega}^{*}-k_{1} e_{\omega}\right)+\widetilde{\tau}_{l}-k_{1} e_{\omega} .
\end{aligned}
$$


Lyapunov function $V_{1}$ is selected as follows:

$$
V_{1}=\frac{1}{2} e_{\omega}^{2}
$$

Then, the derivative of $V_{1}$ can be described as

$$
\begin{aligned}
\dot{V}_{1} & =e_{\omega} \dot{e}_{\omega} \\
& =e_{\omega}\left(\frac{1}{\delta} e_{q}+\frac{\widehat{\delta}}{\delta}\left(\widehat{\tau}_{l}+\dot{\omega}^{*}-k_{1} e_{\omega}\right)+\widetilde{\tau}_{l}-k_{1} e_{\omega}\right) .
\end{aligned}
$$

Step 2. To stabilize the output $q$ axis current of PMSM, the derivative of $e_{q}$ is conducted as follows:

$$
\begin{aligned}
\dot{e}_{q} & =\dot{i}_{q}-\dot{i}_{q}^{*}=-i_{q}-\omega \cdot i_{d}+\gamma \cdot \omega+u_{q}+\Delta_{1}-[\dot{\omega} \\
& \left.+\dot{\widehat{\delta}}\left(\widehat{\tau}_{l}+\dot{\omega}^{*}-k_{1} \cdot e_{\omega}\right)+\widehat{\delta}\left(\dot{\hat{\tau}}_{l}+\ddot{\omega}^{*}-k_{1} \cdot \dot{e}_{\omega}\right)\right] \\
& =-i_{q}-\omega \cdot i_{d}+\gamma \cdot \omega+u_{q}+\Delta_{1}-[\dot{\omega} \\
& \left.+\dot{\widehat{\delta}}\left(\widehat{\tau}_{l}+\dot{\omega}^{*}-k_{1} \cdot e_{\omega}\right)+\widehat{\delta}\left(\dot{\hat{\tau}}_{l}+\ddot{\omega}^{*}-k_{1} \cdot \dot{e}_{\omega}\right)\right] \\
& =-i_{q}-\omega \cdot i_{d}+\widehat{\gamma} \cdot \omega-\tilde{\gamma} \cdot \omega+u_{q}+\Delta_{1}-\dot{\widehat{\delta}}\left(\widehat{\tau}_{l}\right. \\
& \left.+\dot{\omega}^{*}-k_{1} \cdot e_{\omega}\right)-\dot{\omega}-\widehat{\delta}\left(\dot{\hat{\tau}}_{l}+\ddot{\omega}^{*}-k_{1} \cdot \dot{e}_{\omega}\right)=-i_{q} \\
& -\omega \cdot i_{d}+\widehat{\gamma} \cdot \omega-\tilde{\gamma} \cdot \omega+u_{q}+\Delta_{1}-\dot{\hat{\delta}}\left(\widehat{\tau}_{l}+\dot{\omega}^{*}-k_{1}\right. \\
& \left.\cdot e_{\omega}\right)-\dot{e}_{\omega}-\dot{\omega}^{*}-\widehat{\delta}\left(\dot{\widehat{\tau}}_{l}+\ddot{\omega}^{*}\right)+\widehat{\delta} \cdot k_{1}\left(\omega-\dot{\omega}^{*}\right) .
\end{aligned}
$$

By substitution of (12) into (15), we can get

$$
\begin{aligned}
\dot{e}_{q}= & -i_{q}-\omega \cdot i_{d}+\hat{\gamma} \cdot \omega-\tilde{\gamma} \cdot \omega+u_{q}+\Delta_{1} \\
& -\dot{\widehat{\delta}}\left(\widehat{\tau}_{l}+\dot{\omega}^{*}-k_{1} \cdot e_{\omega}\right)-\frac{1}{\delta} e_{q} \\
& -\frac{\widetilde{\delta}}{\delta}\left(\widehat{\tau}_{l}+\dot{\omega}^{*}-k_{1} \cdot e_{\omega}\right)-\widehat{\tau}_{l}+k_{1} \cdot e_{\omega}-\dot{\omega}^{*} \\
& -\widehat{\delta}\left(\dot{\widehat{\tau}}_{l}+\ddot{\omega}^{*}\right)+\widehat{\delta} \cdot k_{1}\left(\omega-\dot{\omega}^{*}\right) .
\end{aligned}
$$

Combined with the mathematical model of PMSM chaotic system, (16) can be further calculated as follows:

$$
\begin{aligned}
& \dot{e}_{q}=-i_{q}-\omega \cdot i_{d}+\widehat{\gamma} \cdot \omega-\tilde{\gamma} \cdot \omega+u_{q}+\Delta_{1} \\
& -\dot{\hat{\delta}}\left(\widehat{\tau}_{l}+\dot{\omega}^{*}-k_{1} \cdot e_{\omega}\right)-\frac{1}{\delta} e_{q} \\
& -\frac{\widetilde{\delta}}{\delta}\left(\widehat{\tau}_{l}+\dot{\omega}^{*}-k_{1} \cdot e_{\omega}\right)-\widehat{\tau}_{l}+k_{r} \cdot e_{\omega}-\dot{\omega}^{*} \\
& -\widehat{\delta}\left(\dot{\hat{\tau}}_{l}+\ddot{\omega}^{*}\right)+\widehat{\delta} \cdot k_{1} \cdot \frac{i_{q}-\omega-\tau_{l}}{\delta}-\widehat{\delta} \cdot k_{1} \cdot \dot{\omega}^{*} \\
& =-i_{q}-\omega \cdot i_{d}+\widehat{\gamma} \cdot \omega-\tilde{\gamma} \cdot \omega+u_{q}+\Delta_{1} \\
& -\dot{\hat{\delta}}\left(\widehat{\tau}_{l}+\dot{\omega}^{*}-k_{1} \cdot e_{\omega}\right)-\frac{1}{\delta} e_{q} \\
& -\frac{\widetilde{\delta}}{\delta}\left(\widehat{\tau}_{l}+\dot{\omega}^{*}-k_{1} \cdot e_{\omega}\right)-\widehat{\tau}_{l}+k_{1} \cdot e_{\omega}-\dot{\omega}^{*}
\end{aligned}
$$

$$
\begin{aligned}
& -\widehat{\delta}\left(\dot{\vec{\tau}}_{l}+\ddot{\omega}^{*}\right)+\frac{\widehat{\delta}}{\delta} \cdot k_{1} \cdot\left(i_{q}-\omega\right)-\widehat{\delta} \cdot k_{1} \cdot \tau_{l}-\widehat{\delta} \\
& \cdot k_{1} \cdot \dot{\omega}^{*} \\
& =-i_{q}-\omega \cdot i_{d}+\widehat{\gamma} \cdot \omega-\tilde{\gamma} \cdot \omega+u_{q}+\Delta_{1} \\
& -\dot{\hat{\delta}}\left(\widehat{\tau}_{l}+\dot{\omega}^{*}-k_{1} \cdot e_{\omega}\right)-\frac{1}{\delta} e_{q} \\
& -\frac{\widetilde{\delta}}{\delta}\left(\widehat{\tau}_{l}+\dot{\omega}^{*}-k_{1} \cdot e_{\omega}\right)-\widetilde{\tau}_{l}+k_{1} \cdot e_{\omega}-\dot{\omega}^{*} \\
& -\widehat{\delta}\left(\dot{\hat{\tau}}_{l}+\ddot{\omega}^{*}\right)+\frac{\widehat{\delta}}{\delta} \cdot k_{1} \cdot\left(i_{q}-\omega\right)-\widehat{\delta} \cdot k_{1} \\
& \cdot\left(\widehat{\tau}_{l}-\widetilde{\tau}_{l}\right)-\widehat{\delta} \cdot k_{1} \cdot \dot{\omega}^{*} \\
& =-i_{q}-\omega \cdot i_{d}+\widehat{\gamma} \cdot \omega-\tilde{\gamma} \cdot \omega+u_{q}+\Delta_{1} \\
& -\dot{\hat{\delta}}\left(\widehat{\tau}_{l}+\dot{\omega}^{*}-k_{1} \cdot e_{\omega}\right)-\frac{1}{\delta} e_{q} \\
& -\frac{\widetilde{\delta}}{\delta}\left(\widehat{\tau}_{l}+\dot{\omega}^{*}-k_{1} \cdot e_{\omega}\right)-\widehat{\tau}_{l}+k_{1} \cdot e_{\omega}-\dot{\omega}^{*} \\
& -\widehat{\delta}\left(\dot{\vec{\tau}}_{l}+\ddot{\omega}^{*}\right)+\frac{\widehat{\delta}}{\delta} \cdot k_{1} \cdot\left(i_{q}-\omega\right)-\widehat{\delta} \cdot k_{1} \cdot \widehat{\tau}_{l}+\widehat{\delta} \\
& \cdot k_{1} \cdot \tilde{\tau}_{l}-\widehat{\delta} \cdot k_{1} \cdot \dot{\omega}^{*} \text {. }
\end{aligned}
$$

By substitution of $\widehat{\delta}=\widetilde{\delta}+\delta$ into (17), the following equation can be obtained:

$$
\begin{aligned}
& \dot{e}_{q}=-i_{q}-\omega \cdot i_{d}+\hat{\gamma} \cdot \omega-\tilde{\gamma} \cdot \omega+u_{q}+\Delta_{1} \\
& -\dot{\hat{\delta}}\left(\widehat{\tau}_{l}+\dot{\omega}^{*}-k_{1} e_{\omega}\right)-\frac{1}{\delta} e_{q} \\
& -\frac{\widetilde{\delta}}{\delta}\left(\widehat{\tau}_{l}+\dot{\omega}^{*}-k_{1} e_{\omega}\right)-\widetilde{\tau}_{l}+k_{1} e_{\omega}-\dot{\omega}^{*} \\
& -\widehat{\delta}\left(\dot{\widehat{\tau}}_{l}+\ddot{\omega}^{*}\right)+\frac{\delta+\widetilde{\delta}}{\delta} \cdot k_{1} \cdot\left(i_{q}-\omega\right)-\widehat{\delta} \cdot k_{1} \cdot \widehat{\tau}_{l} \\
& +\widehat{\delta} \cdot k_{1} \cdot \widetilde{\tau}_{l}-\widehat{\delta} \cdot k_{1} \cdot \dot{\omega}^{*} \\
& =-i_{q}-\omega \cdot i_{d}+\hat{\gamma} \cdot \omega-\tilde{\gamma} \cdot \omega+u_{q}+\Delta_{1} \\
& -\dot{\hat{\delta}}\left(\widehat{\tau}_{l}+\dot{\omega}^{*}-k_{1} e_{\omega}\right)-\frac{1}{\delta} e_{q} \\
& -\frac{\widetilde{\delta}}{\delta}\left(\widehat{\tau}_{l}+\dot{\omega}^{*}-k_{1} e_{\omega}\right)-\widetilde{\tau}_{l}+k_{1} e_{\omega}-\dot{\omega}^{*} \\
& -\widehat{\delta}\left(\dot{\hat{\tau}}_{l}+\ddot{\omega}^{*}\right)+k_{1} \cdot\left(i_{q}-\omega\right)+\frac{\widetilde{\delta}}{\delta} \cdot k_{1} \cdot\left(i_{q}-\omega\right) \\
& -\widehat{\delta} \cdot k_{1} \cdot \widehat{\tau}_{l}+\widehat{\delta} \cdot k_{1} \cdot \widetilde{\tau}_{l}-\widehat{\delta} \cdot k_{1} \cdot \dot{\omega}^{*}
\end{aligned}
$$

Lyapunov function $V_{2}$ is chosen as follows:

$$
V_{2}=V_{1}+\frac{1}{2} e_{q}^{2}
$$


Then, the derivative of $V_{2}$ can be described as

$$
\begin{aligned}
\dot{V}_{2} & =\dot{V}_{1}+e_{q} \dot{e}_{q}=e_{\omega}\left(\frac{1}{\delta} e_{q}+\frac{\widehat{\delta}}{\delta}\left(\widehat{\tau}_{l}+\dot{\omega}^{*}-k_{1} e_{\omega}\right)+\widetilde{\tau}_{l}\right. \\
& \left.-k_{1} e_{\omega}\right)+e_{q}\left(-i_{q}-\omega \cdot i_{d}+\widehat{\gamma} \cdot \omega-\widetilde{\gamma} \cdot \omega+u_{q}\right. \\
& +\Delta_{1}-\dot{\hat{\delta}}\left(\widehat{\tau}_{l}+\dot{\omega}^{*}-k_{1} e_{\omega}\right)-\frac{1}{\delta} e_{q} \\
& -\frac{\widehat{\delta}}{\delta}\left(\widehat{\tau}_{l}+\dot{\omega}^{*}-k_{1} e_{\omega}\right)-\widetilde{\tau}_{l}+k_{1} e_{\omega}-\dot{\omega}^{*} \\
& -\widehat{\delta}\left(\dot{\hat{\tau}}_{l}+\ddot{\omega}^{*}\right)+k_{1} \cdot\left(i_{q}-\omega\right)+\frac{\widetilde{\delta}_{\delta}}{\delta} k_{1} \cdot\left(i_{q}-\omega\right) \\
& \left.-\widehat{\delta} \cdot k_{1} \cdot \widehat{\tau}_{l}+\widehat{\delta} \cdot k_{1} \cdot \widetilde{\tau}_{l}-\widehat{\delta} \cdot k_{1} \cdot \dot{\omega}^{*}\right)
\end{aligned}
$$

The first control variable is selected as

$$
u_{q}=u_{q s}+u_{q r}
$$

where $u_{q s}$ and $u_{q r}$ are the model compensation and robust control inputs, respectively.

Then, $u_{q s}$ and $u_{q r}$ can be, respectively, chosen as

$$
\begin{aligned}
u_{q s}= & -k_{2} \cdot e_{q}+i_{q}+\omega \cdot i_{d}-\widehat{\gamma} \cdot \omega \\
& +\dot{\widehat{\delta}}\left(\widehat{\tau}_{l}+\dot{\omega}^{*}-k_{1} e_{\omega}\right)+\dot{\omega}^{*}+\widehat{\delta}\left(\dot{\hat{\tau}}_{l}+\ddot{\omega}^{*}\right)+\widehat{\delta} \\
& \cdot k_{1} \cdot \widehat{\tau}_{l}+\widehat{\delta} \cdot k_{r} \cdot \dot{\omega}^{*}-k_{1}\left(i_{q}-\omega\right), \\
u_{q r}= & -\frac{d_{1}^{2}(\mathbf{x})}{4 \varepsilon_{1}} e_{q},
\end{aligned}
$$

where $k_{2}$ is another positive control gain and $\varepsilon_{1}$ is a positive number chosen arbitrarily.

By substitution of (21) and (22) into (20), we can acquire

$$
\begin{aligned}
\dot{V}_{2} & =\dot{V}_{1}+e_{q} \dot{e}_{q}=e_{\omega}\left(\frac{1}{\delta} e_{q}+\frac{\widehat{\delta}}{\delta}\left(\widehat{\tau}_{l}+\dot{\omega}^{*}-k_{1} e_{\omega}\right)+\widetilde{\tau}_{l}\right. \\
& \left.-k_{1} e_{\omega}\right)+e_{q}\left(-k_{2} e_{q}-\frac{1}{\delta} \cdot e_{q}+k_{1} e_{\omega}-\tilde{\gamma} \cdot \omega+u_{q r}\right. \\
& +\Delta_{1}-\frac{\widetilde{\delta}}{\delta}\left(\left(\widehat{\tau}_{l}+\dot{\omega}^{*}-k_{1} e_{\omega}\right)-k_{1} \cdot\left(i_{q}-\omega\right)\right) \\
& \left.+\widetilde{\tau}_{l}\left(\widehat{\delta} \cdot k_{1}-1\right)\right)=e_{\omega}\left(\frac{1}{\delta} e_{q}\right. \\
& \left.+\frac{\widehat{\delta}}{\delta}\left(\widehat{\tau}_{l}+\dot{\omega}^{*}-k_{1} e_{\omega}\right)+\widetilde{\tau}_{l}-k_{1} e_{\omega}\right)+e_{q}\left(-k_{2} e_{q}\right. \\
& -\frac{1}{\delta} \cdot e_{q}+k_{1} e_{\omega}-\tilde{\gamma} \cdot \omega-\frac{d_{1}^{2}(\mathbf{x})}{4 \varepsilon_{1}} e_{q}+\Delta_{1}
\end{aligned}
$$

$$
\begin{aligned}
& -\frac{\widetilde{\delta}}{\delta}\left(\left(\widehat{\tau}_{l}+\dot{\omega}^{*}-k_{1} e_{\omega}\right)-k_{1} \cdot\left(i_{q}-\omega\right)\right) \\
& \left.+\widetilde{\tau}_{l}\left(\widehat{\delta} \cdot k_{1}-1\right)\right) .
\end{aligned}
$$

Additionally,

$$
\begin{aligned}
& e_{q}\left(-\frac{d_{1}^{2}(\mathbf{x})}{4 \varepsilon_{1}} e_{q}+\Delta_{1}\right)=-\frac{d_{1}^{2}(\mathbf{x})}{4 \varepsilon_{1}} e_{q}^{2}+\Delta_{1} e_{q} \\
& \leq-\frac{d_{1}^{2}(\mathbf{x})}{4 \varepsilon_{1}} e_{q}^{2}+d_{1}(\mathbf{x}) f_{1 \max }\left|e_{q}\right| \\
& \quad=-\left(\frac{d_{1}(\mathbf{x})\left|e_{q}\right|}{2 \sqrt{\varepsilon_{1}}}-\sqrt{\varepsilon_{1}} f_{1 \max }\right)^{2}+\varepsilon_{1} f_{1 \max }^{2} .
\end{aligned}
$$

Step 3. Differentiating the tracking error $e_{d}$ of $d$ axis current $i_{d}$, we can get

$$
\dot{e}_{d}=\dot{i}_{d}-\dot{i}_{d}^{*}=-i_{d}+\omega \cdot i_{q}+u_{d}+\Delta_{2}-\dot{i}_{d}^{*} .
$$

Lyapunov function $V_{3}$ is chosen as

$$
V_{3}=V_{2}+\frac{1}{2} e_{d}^{2}
$$

Then, the derivative of $V_{3}$ can be represented as

$$
\begin{aligned}
\dot{V}_{3} & =\dot{V}_{2}+e_{d} \dot{e}_{d} \\
& =\dot{V}_{2}+e_{d}\left(-i_{d}+\omega \cdot i_{q}+u_{d}+\Delta_{2}-\dot{i}_{d}^{*}\right) .
\end{aligned}
$$

In terms of (28), $d$ axis output stator voltage $u_{d}$ can be calculated:

$$
u_{d}=u_{d s}+u_{d r}
$$

where

$$
\begin{aligned}
& u_{d s}=-k_{3} \cdot e_{d}+i_{d}-\omega \cdot i_{q}+i_{d}^{*} \\
& u_{d r}=-\frac{d_{2}^{2}(\mathbf{x})}{4 \varepsilon_{2}} e_{d}
\end{aligned}
$$

where $k_{3}$ is the positive control gain and $\varepsilon_{2}$ is a positive number chosen arbitrarily.

By substitution of (30) and (31) into (26) and (28), respectively, the following equations can be acquired:

$$
\begin{aligned}
\dot{e}_{d} & =-k_{3} \cdot e_{d}+u_{d r}+\Delta_{2}=-k_{3} \cdot e_{d}-\frac{d_{2}^{2}(\mathbf{x})}{4 \varepsilon_{2}} e_{d}+\Delta_{2}, \\
\dot{V}_{3} & =\dot{V}_{2}+e_{d} \dot{e}_{d} \\
& =\dot{V}_{2}+e_{d}\left(-k_{3} \cdot e_{d}-\frac{d_{2}^{2}(\mathbf{x})}{4 \varepsilon_{2}} e_{d}+\Delta_{2}\right)
\end{aligned}
$$




$$
\begin{aligned}
& e_{d}\left(-\frac{d_{2}^{2}(\mathbf{x})}{4 \varepsilon_{2}} e_{d}+\Delta_{2}\right)=-\frac{d_{2}^{2}(\mathbf{x})}{4 \varepsilon_{2}} e_{d}^{2}+\Delta_{2} e_{d} \\
& \quad \leq-\frac{d_{2}^{2}(\mathbf{x})}{4 \varepsilon_{2}} e_{d}^{2}+d_{2}(\mathbf{x}) f_{2 \max }\left|e_{d}\right| \\
& \quad=-\left(\frac{d_{2}(\mathbf{x})\left|e_{d}\right|}{2 \sqrt{\varepsilon_{2}}}-\sqrt{\varepsilon_{2}} f_{2 \max }\right)^{2}+\varepsilon_{2} f_{2 \max }^{2} .
\end{aligned}
$$

Step 4. Lyapunov function $V$ of PMSM chaotic system with fully unknown parameters and external disturbances is selected as follows:

$$
V=V_{3}+\frac{1}{2 \theta_{1}} \widetilde{\tau}_{l}^{2}+\frac{1}{2 \theta_{2}} \widetilde{\gamma}^{2}+\frac{1}{2 \delta \cdot \theta_{3}} \widetilde{\delta}^{2},
$$

where $\theta_{1}, \theta_{2}$, and $\theta_{3}$ represent positive adaptive gains.

Combined with equations $\dot{\widetilde{\delta}}=\dot{\hat{\delta}}, \dot{\tilde{\gamma}}=\dot{\hat{\gamma}}$, and $\dot{\tilde{\tau}}_{l}=\dot{\hat{\tau}}_{l}$, derivative of selected Lyapunov function $V$ can be calculated as follows:

$$
\begin{aligned}
& \dot{V}=\dot{V}_{3}+\frac{\widetilde{\tau}_{l}}{\theta_{1}} \dot{\widehat{\tau}}_{l}+\frac{\tilde{\gamma}}{\theta_{2}} \dot{\hat{\gamma}}+\frac{\widetilde{\delta}}{\delta \cdot \theta_{3}} \dot{\hat{\delta}}=\dot{V}_{2}+e_{d}\left(-k_{3} \cdot e_{d}\right. \\
& \left.+u_{d r}+\Delta_{2}\right)=\dot{V}_{1}+e_{q}\left(-k_{2} e_{q}-\frac{1}{\delta} \cdot e_{q}+k_{1} e_{\omega}-\tilde{\gamma}\right. \\
& \cdot \omega+u_{q r}+\Delta_{1} \\
& -\frac{\widetilde{\delta}}{\delta}\left(\left(\widehat{\tau}_{l}+\dot{\omega}^{*}-k_{1} e_{\omega}\right)-k_{1} \cdot\left(i_{q}-\omega\right)\right) \\
& \left.+\tilde{\tau}_{l}\left(\widehat{\delta} \cdot k_{1}-1\right)\right)+e_{d}\left(-k_{3} \cdot e_{d}+u_{d r}+\Delta_{2}\right) \\
& =e_{\omega}\left(\frac{1}{\delta} e_{q}+\frac{\widehat{\delta}}{\delta}\left(\widehat{\tau}_{l}+\dot{\omega}^{*}-k_{1} e_{\omega}\right)+\widetilde{\tau}_{l}-k_{1} e_{\omega}\right) \\
& +e_{q}\left(-k_{2} e_{q}-\frac{1}{\delta} \cdot e_{q}+k_{1} e_{\omega}-\tilde{\gamma} \cdot \omega+u_{q r}+\Delta_{1}\right. \\
& -\frac{\widetilde{\delta}}{\delta}\left(\left(\widehat{\tau}_{l}+\dot{\omega}^{*}-k_{1} e_{\omega}\right)-k_{1} \cdot\left(i_{q}-\omega\right)\right) \\
& \left.+\widetilde{\tau}_{l}\left(\widehat{\delta} \cdot k_{1}-1\right)\right)+e_{d}\left(-k_{3} \cdot e_{d}+u_{d r}+\Delta_{2}\right)=\frac{1}{\delta} \\
& \cdot e_{q} e_{\omega}+\frac{\widetilde{\delta}}{\delta}\left(\widehat{\tau}_{l}+\dot{\omega}^{*}-k_{1} e_{\omega}\right) \cdot e_{\omega}+\widetilde{\tau}_{l} e_{\omega}-k_{1} e_{\omega}^{2} \\
& -k_{3} e_{d}^{2}-k_{2} e_{q}^{2}-\tilde{\gamma} \omega e_{q}-\frac{1}{\delta} e_{q}^{2}+e_{q}\left(u_{q r}+\Delta_{1}\right) \\
& +e_{d}\left(u_{d r}+\Delta_{2}\right)-\frac{\widetilde{\delta}}{\delta}\left(\left(\widehat{\tau}_{l}+\dot{\omega}^{*}-k_{1} e_{\omega}\right)\right. \\
& \left.-k_{1}\left(i_{q}-\omega\right)\right) \cdot e_{q}+\widetilde{\tau}_{l}\left(\widehat{\delta} \cdot k_{1}-1\right) e_{q}+k_{1} e_{\omega} e_{q} \\
& +\frac{\tilde{\tau}_{l}}{\theta_{1}} \dot{\hat{\tau}}_{l}+\frac{\tilde{\gamma}}{\theta_{2}} \dot{\hat{\gamma}}+\frac{\widetilde{\delta}}{\delta \theta_{3}} \dot{\hat{\delta}}=\frac{1}{\delta} e_{q} e_{\omega}+k_{1} e_{\omega} e_{q}
\end{aligned}
$$

$$
\begin{aligned}
& +\frac{\tilde{\delta}}{\delta}\left[\left(\widehat{\tau}_{l}+\dot{\omega}^{*}-k_{1} e_{\omega}\right) e_{\omega}-\left(\widehat{\tau}_{l}+\dot{\omega}^{*}-k_{1} e_{\omega}\right) e_{q}\right. \\
& \left.+k_{1}\left(i_{q}-\omega\right) e_{q}+\frac{\dot{\hat{\delta}}}{\theta_{3}}\right]-k_{1} e_{\omega}^{2}-k_{2} e_{d}^{2}-k_{3} e_{q}^{2} \\
& +\widetilde{\tau}_{l}\left[e_{\omega}-e_{q}+\widehat{\delta} k_{1} e_{q}+\frac{\dot{\hat{\tau}}_{l}}{\theta_{1}}\right]+\widetilde{\gamma}\left[-\omega e_{q}+\frac{\dot{\hat{\gamma}}}{\theta_{2}}\right] \\
& -\frac{1}{\delta} e_{q}^{2}+e_{q}\left(-\frac{d_{1}^{2}(\mathbf{x})}{4 \varepsilon_{1}} e_{q}+\Delta_{1}\right)+e_{d}\left(-\frac{d_{2}^{2}(\mathbf{x})}{4 \varepsilon_{2}} e_{d}\right. \\
& \left.+\Delta_{2}\right) .
\end{aligned}
$$

In terms of (36), the adaptive laws of unknown parameters $\delta$, $\gamma$, and $\tau_{l}$ can be selected, respectively, as follows:

$$
\begin{aligned}
\dot{\hat{\delta}} & =-\theta_{3}\left[\left(\widehat{\tau}_{l}+\dot{\omega}^{*}-k_{1} e_{\omega}\right) \cdot\left(e_{\omega}-e_{q}\right)\right. \\
& \left.+k_{1}\left(i_{q}-\omega\right) e_{q}\right], \\
\dot{\hat{\gamma}} & =\theta_{2} \omega e_{q}, \\
\dot{\widehat{\tau}}_{l} & =-\theta_{1}\left[e_{\omega}-e_{q}+\widehat{\delta} k_{1} e_{q}\right] .
\end{aligned}
$$

By substitution of (37), (38), and (39) into (36), (36) can be simplified as follows:

$$
\begin{aligned}
\dot{V}= & \frac{1}{\delta} e_{q} e_{\omega}+k_{1} e_{q} e_{\omega}-k_{1} e_{\omega}^{2}-k_{3} e_{d}^{2}-k_{2} e_{q}^{2}-\frac{1}{\delta} e_{q}^{2} \\
& +e_{q}\left(-\frac{d_{1}^{2}(\mathbf{x})}{4 \varepsilon_{1}} e_{q}+\Delta_{1}\right) \\
& +e_{d}\left(-\frac{d_{2}^{2}(\mathbf{x})}{4 \varepsilon_{2}} e_{d}+\Delta_{2}\right) .
\end{aligned}
$$

\subsection{Stability Analysis}

Theorem 4. For PMSM chaotic system model (3) with fully unknown parameters and external disturbances, design of adaptive control laws (37), (38), and (40) and selection of suitable controller gains $k_{1}, k_{2}, k_{3}, \varepsilon_{1}$, and $\varepsilon_{2}$ and adaptive gains $\theta_{1}, \theta_{2}$, and $\theta_{3}$, the proposed adaptive robust backstepping controllers (21) and (29) can ensure the tracking error signals (5), (7), and (8) of PMSM chaotic systems are asymptotically stable. That is to say, PMSM chaotic system can run out of chaos quickly through the proposed controllers (21) and (29) and track the given reference signals.

Through stability analysis, we want to verify the correctness of the theorem. 
According to (40), new expression can be obtained as follows through some mathematical computations:

$$
\begin{aligned}
\dot{V}= & \frac{1}{\delta} e_{q} e_{\omega}+k_{1} e_{q} e_{\omega}-k_{1} e_{\omega}^{2}-k_{3} e_{d}^{2}-k_{2} e_{q}^{2}-\frac{1}{\delta} e_{q}^{2} \\
& +e_{q}\left(-\frac{d_{1}^{2}(\mathbf{x})}{4 \varepsilon_{1}} e_{q}+\Delta_{1}\right)+e_{d}\left(-\frac{d_{2}^{2}(\mathbf{x})}{4 \varepsilon_{2}} e_{d}+\Delta_{2}\right) \\
= & -\frac{1}{2 \delta}\left(e_{\omega}-e_{q}\right)^{2}+\frac{1}{2 \delta} e_{\omega}^{2}+\frac{1}{2 \delta} e_{q}^{2}-\frac{k_{1}}{2}\left(e_{\omega}-e_{q}\right)^{2} \\
& +\frac{k_{1}}{2} e_{\omega}^{2}+\frac{k_{1}}{2} e_{q}^{2}-k_{1} e_{\omega}^{2}-k_{3} e_{d}^{2}-k_{2} e_{q}^{2}-\frac{1}{\delta} e_{q}^{2} \\
& +e_{q}\left(-\frac{d_{1}^{2}(\mathbf{x})}{4 \varepsilon_{1}} e_{q}+\Delta_{1}\right)+e_{d}\left(-\frac{d_{2}^{2}(\mathbf{x})}{4 \varepsilon_{2}} e_{d}+\Delta_{2}\right) \\
= & -\frac{1}{2 \delta}\left(e_{\omega}-e_{q}\right)^{2}-\frac{k_{1}}{2}\left(e_{\omega}-e_{q}\right)^{2}-k_{3} e_{d}^{2} \\
& +\left(\frac{1}{2 \delta}+\frac{k_{1}}{2}-k_{1}\right) e_{\omega}^{2}+\left(\frac{1}{2 \delta}+\frac{k_{1}}{2}-k_{2}-\frac{1}{\delta}\right) e_{q}^{2} \\
& +e_{q}\left(-\frac{d_{1}^{2}(\mathbf{x})}{4 \varepsilon_{1}} e_{q}+\Delta_{1}\right) \\
& +e_{d}\left(-\frac{d_{2}^{2}(\mathbf{x})}{4 \varepsilon_{2}} e_{d}+\Delta_{2}\right) .
\end{aligned}
$$

Appropriate controller gains $k_{1}$ and $k_{2}$ are selected as follows:

$$
\begin{aligned}
\left(\frac{1}{2 \delta}+\frac{k_{1}}{2}-k_{1}\right) & <0, \\
\left(\frac{1}{2 \delta}+\frac{k_{1}}{2}-k_{2}-\frac{1}{\delta}\right) & <0 .
\end{aligned}
$$

Equation (42) can be replaced by the following:

$$
\begin{aligned}
k_{1} & >\frac{1}{\delta}, \\
k_{1}-2 k_{2} & <\frac{1}{\delta} .
\end{aligned}
$$

Then, by substitution of (43) into (41), we can obtain

$$
\begin{aligned}
\dot{V}= & -\frac{1}{2 \delta}\left(e_{\omega}-e_{q}\right)^{2}-\frac{k_{1}}{2}\left(e_{\omega}-e_{q}\right)^{2}-k_{3} e_{d}^{2} \\
& +\left(\frac{1}{2 \delta}+\frac{k_{1}}{2}-k_{1}\right) e_{\omega}^{2}+\left(\frac{1}{2 \delta}+\frac{k_{1}}{2}-k_{2}-\frac{1}{\delta}\right) e_{q}^{2} \\
& +e_{q}\left(-\frac{d_{1}^{2}(\mathbf{x})}{4 \varepsilon_{1}} e_{q}+\Delta_{1}\right) \\
& +e_{d}\left(-\frac{d_{2}^{2}(\mathbf{x})}{4 \varepsilon_{2}} e_{d}+\Delta_{2}\right)
\end{aligned}
$$

$$
\begin{aligned}
\leq & -\frac{1}{2 \delta}\left(e_{\omega}-e_{q}\right)^{2}-\frac{k_{1}}{2}\left(e_{\omega}-e_{q}\right)^{2}-k_{3} e_{d}^{2}-k_{4} e_{\omega}^{2} \\
& -k_{5} e_{q}^{2}-\left(\frac{d_{1}(\mathbf{x})\left|e_{q}\right|}{2 \sqrt{\varepsilon_{1}}}-\sqrt{\varepsilon_{1}} f_{1 \max }\right)^{2}+\varepsilon_{1} f_{1 \max }^{2} \\
& -\left(\frac{d_{2}(\mathbf{x})\left|e_{d}\right|}{2 \sqrt{\varepsilon_{2}}}-\sqrt{\varepsilon_{2}} f_{2 \max }\right)^{2}+\varepsilon_{2} f_{2 \max }^{2} \\
\leq & -\frac{1}{2 \delta}\left(e_{\omega}-e_{q}\right)^{2}-\frac{k_{1}}{2}\left(e_{\omega}-e_{q}\right)^{2}-k_{3} e_{d}^{2}-k_{4} e_{\omega}^{2} \\
& -k_{5} e_{q}^{2}-\left(\frac{d_{1}(\mathbf{x})\left|e_{q}\right|}{2 \sqrt{\varepsilon_{1}}}-\sqrt{\varepsilon_{1}} f_{1 \max }\right)^{2} \\
& -\left(\frac{d_{2}(\mathbf{x})\left|e_{d}\right|}{2 \sqrt{\varepsilon_{2}}}-\sqrt{\varepsilon_{2}} f_{2 \max }\right)^{2}+\varepsilon_{0},
\end{aligned}
$$

where $k_{4}=-\left(1 / 2 \delta+k_{1} / 2-k_{1}\right) \geq 0, k_{5}=-\left(1 / 2 \delta+k_{1} / 2-\right.$ $\left.k_{2}-1 / \delta\right) \geq 0$, and $\varepsilon_{0}=\varepsilon_{1} f_{1 \max }^{2}+\varepsilon_{2} f_{2 \max }^{2}$.

Let

$$
\begin{aligned}
W(i(t))= & -\frac{1}{2 \delta}\left(e_{\omega}-e_{q}\right)^{2}-\frac{k_{1}}{2}\left(e_{\omega}-e_{q}\right)^{2}-k_{3} e_{d}^{2} \\
& -k_{4} e_{\omega}^{2}-k_{5} e_{q}^{2} \\
& -\left(\frac{d_{1}(\mathbf{x})\left|e_{q}\right|}{2 \sqrt{\varepsilon_{1}}}-\sqrt{\varepsilon_{1}} f_{1 \max }\right)^{2} \\
& -\left(\frac{d_{2}(\mathbf{x})\left|e_{d}\right|}{2 \sqrt{\varepsilon_{2}}}-\sqrt{\varepsilon_{2}} f_{2 \max }\right)^{2}+\varepsilon_{0},
\end{aligned}
$$

where $i(t)=\left(e_{\omega}, e_{q}, e_{d}\right)$.

By integration of (45), we can get

$$
\begin{aligned}
& \int_{t_{0}}^{t} W(i(t)) d t=-\int_{t_{0}}^{t} \dot{V}(i(t)) d t \Longrightarrow \\
& \int_{t_{0}}^{t} W(i(t)) d t=V\left(t_{0}\right)-V(t) .
\end{aligned}
$$

Since $V\left(t_{0}\right)$ is bounded and $V(t)$ is bounded and nonincreasing, hence

$$
\lim _{t \rightarrow \infty} \int_{t_{0}}^{t} W(i(t)) d t<\infty
$$

Moreover, $W(i(t))$ is uniform continuous and $\dot{W}(i(t))$ is bounded. In accordance with Barbalat's Lemma, the following equation can be obtained:

$$
\lim _{t \rightarrow \infty} W(i(t))=0 .
$$

Apparently, through selection of suitable controller gains $k_{1}$, $k_{2}, k_{3}, \varepsilon_{1}$, and $\varepsilon_{2}, V$ can be ensured to be negative definite.

The above derivation has proved that the selected suitable controller gains $k_{1}, k_{2}, k_{3}, \varepsilon_{1}$, and $\varepsilon_{2}$ and adaptive gains $\theta_{1}, \theta_{2}$, 


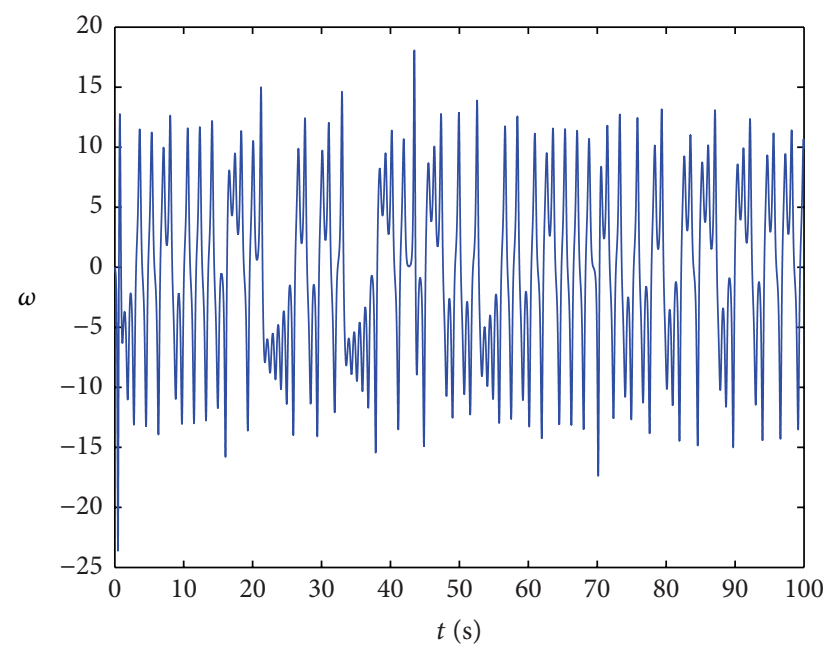

Figure 2: The $\omega$ curve of PMSM chaotic system with no control inputs $u_{d}$ and $u_{q}$.

and $\theta_{3}$ can make the inequalities of $V \geq 0$ and $\dot{V}<0$ hold. In addition, equation of $V=0$ is not satisfied until $e_{\omega}=e_{q}=$ $e_{d}=\widetilde{\tau}_{l}=\tilde{\gamma}=\widetilde{\delta}=0$. In summary, PMSM chaotic system is globally asymptotically stable at the equilibrium point of $\left(e_{\omega}, e_{q}, e_{d}\right)=(0,0,0)$.

\section{Numerical Simulation and Discussions}

In order to illustrate the superiority of the proposed approach adequately, the simulation is carried out in MATLAB environment for three cases under the initial condition of $\left(\omega, i_{q}, i_{d}\right)=(0,0,0)$. Let $\left(\omega, i_{q}, i_{d}\right)=\left(x_{1}, x_{2}, x_{3}\right)$ and the control parameters are selected as $k_{1}=10, k_{2}=30000, k_{3}=5$, and $\varepsilon_{1}=\varepsilon_{2}=0.01$; the adaptive gains are chosen as $\theta_{1}=6.2$, $\theta_{2}=100$, and $\theta_{3}=0.06$. The simulation time is chosen as $100 \mathrm{~s}$ and the designed controller is put into effect at the time of $20 \mathrm{~s}$.

4.1. Test-I. The PMSM chaotic system is tested with the parameters $\delta=0.2, \gamma=50$, and $\tau_{l}=3.2$. In order to be consistent with the reality better, we assume that the three parameters of PMSM chaotic system are all unknown with the initial condition of $\left(\delta, \gamma, \tau_{l}\right)=(0,0,0)$, and the expected reference signals are set as $\omega^{*}=10$ and $i_{d}^{*}=1$. Furthermore, the external disturbances $\Delta_{1}(\mathbf{x}, t)=20 x_{3} \sin (5 t)$ and $\Delta_{2}(\mathbf{x}, t)=10 \sin (5 t)$ are injected into the PMSM chaotic system. The simulation results given in Figures 2-4 apparently show PMSM runs in a chaotic state with no control inputs. Therefore, introduction of the presented control approach to suppress chaos in PMSM system will be of great importance and necessity. Figures 5-12 show that the proposed controller is utilized to control the PMSM chaotic system, where Figures 5-7 display the curves of state variables changing over time for PMSM chaotic system, which demonstrate the PMSM system stays away from the previous chaotic state when the designed controller is added to PMSM chaotic system, and track the desired signals accurately and rapidly. Furthermore,

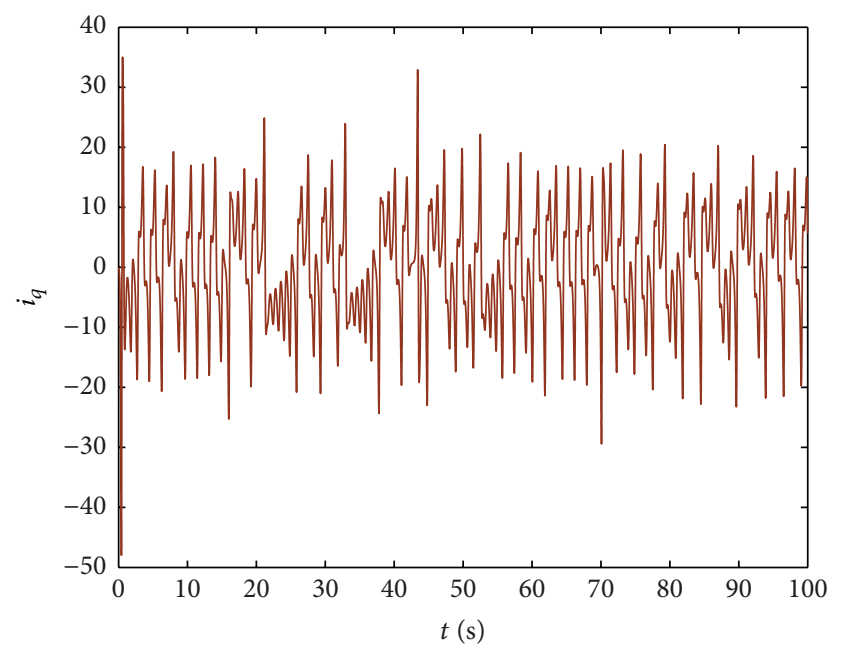

FIGURE 3: The $i_{q}$ curve of PMSM chaotic system with no control inputs $u_{d}$ and $u_{q}$.

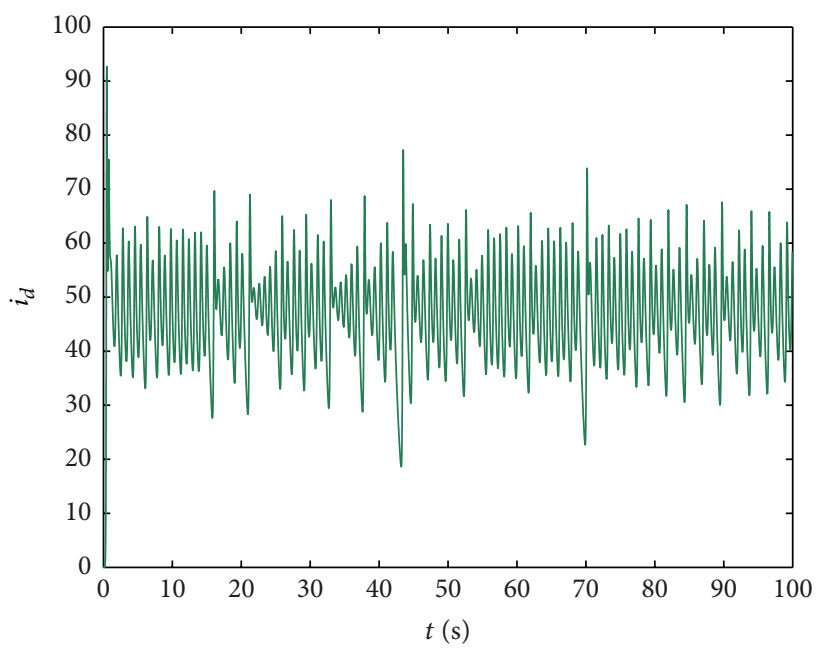

FIgURE 4: The $i_{d}$ curve of PMSM chaotic system with no control inputs $u_{d}$ and $u_{q}$.

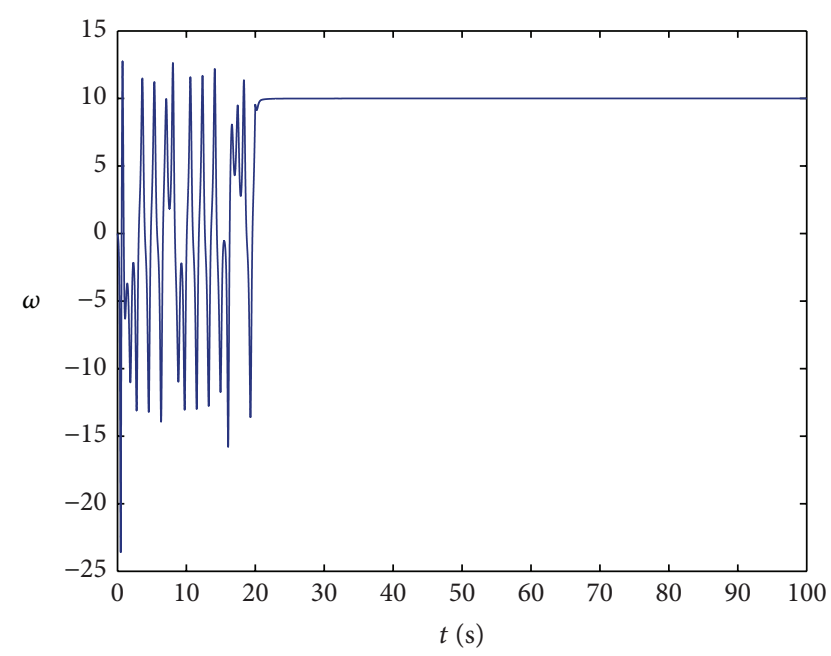

FIGURE 5: The $\omega$ curve of PMSM chaotic system added the controller inputs $u_{d}$ and $u_{q}$. 


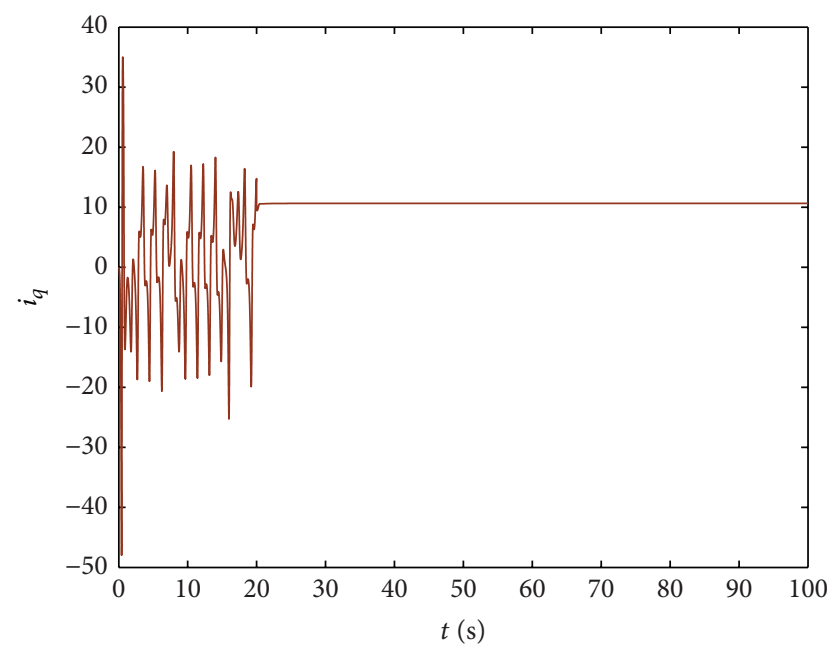

Figure 6: The $i_{q}$ curve of PMSM chaotic system added the controller inputs $u_{d}$ and $u_{q}$.

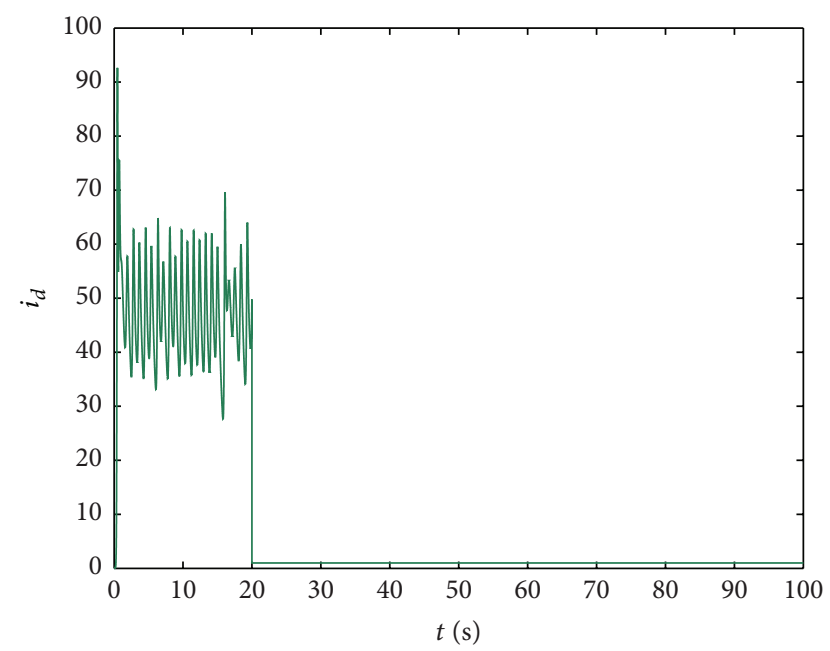

FIGURE 7: The $i_{d}$ curve of PMSM chaotic system added the controller inputs $u_{d}$ and $u_{q}$.

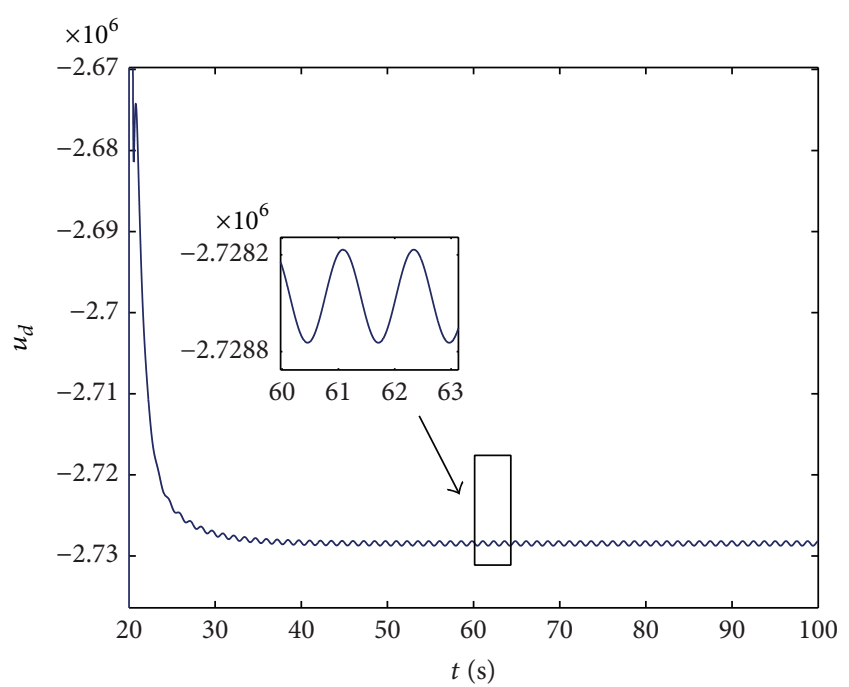

FIGURE 8: The controller input $u_{d}$.

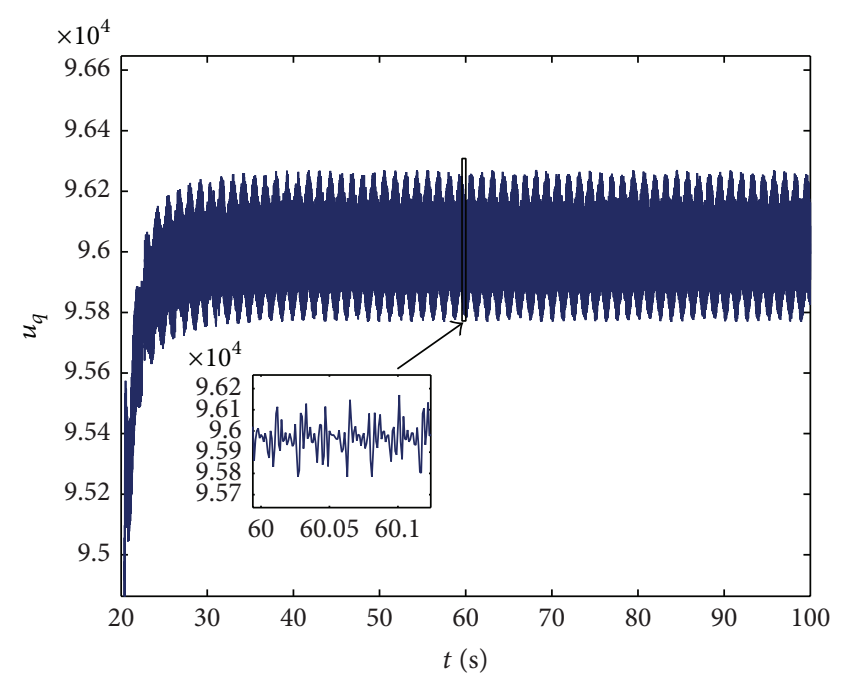

FIgURE 9: The controller input $u_{q}$.

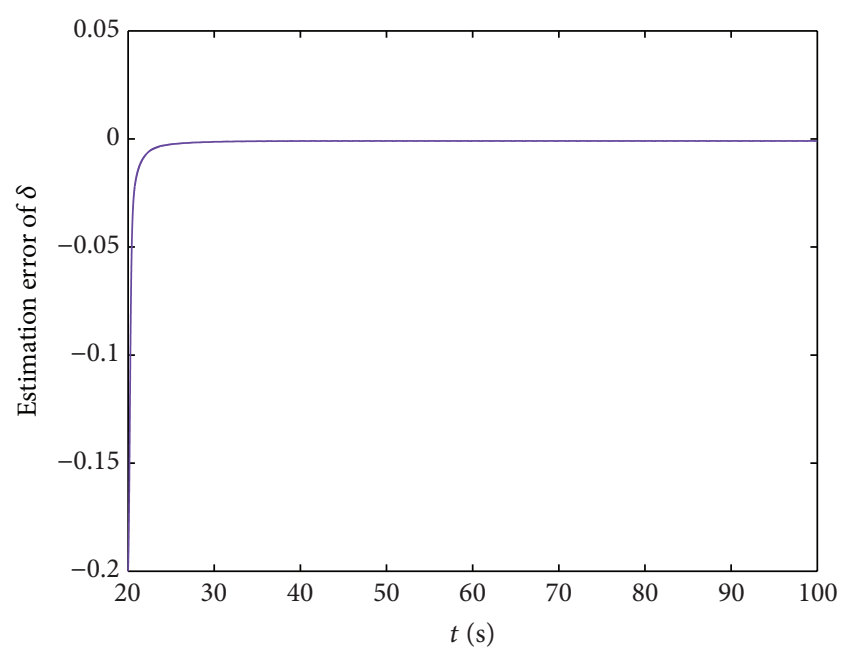

FIGURE 10: The $\widetilde{\delta}$ curve of estimation error of $\delta$.

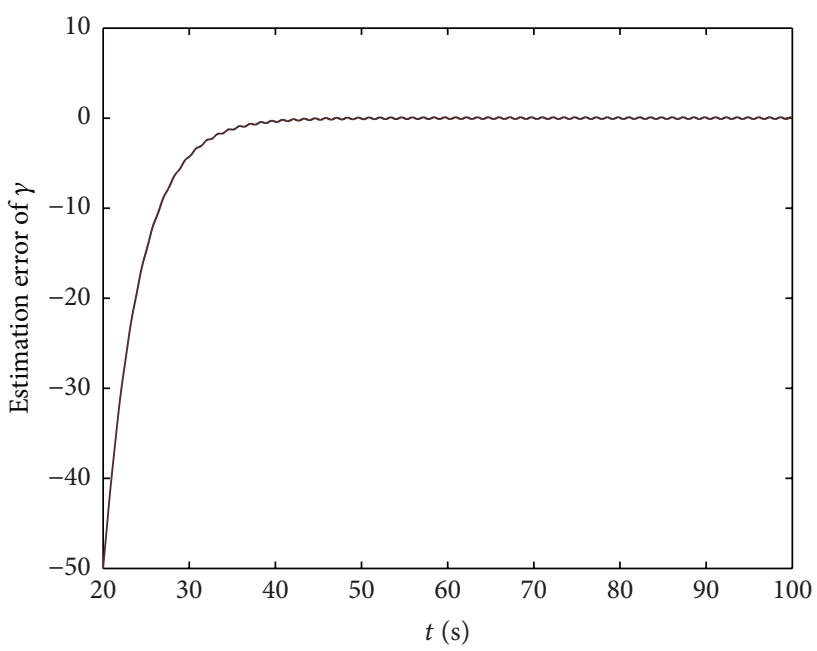

FIGURE 11: The $\tilde{\gamma}$ curve of estimation error of $\gamma$. 


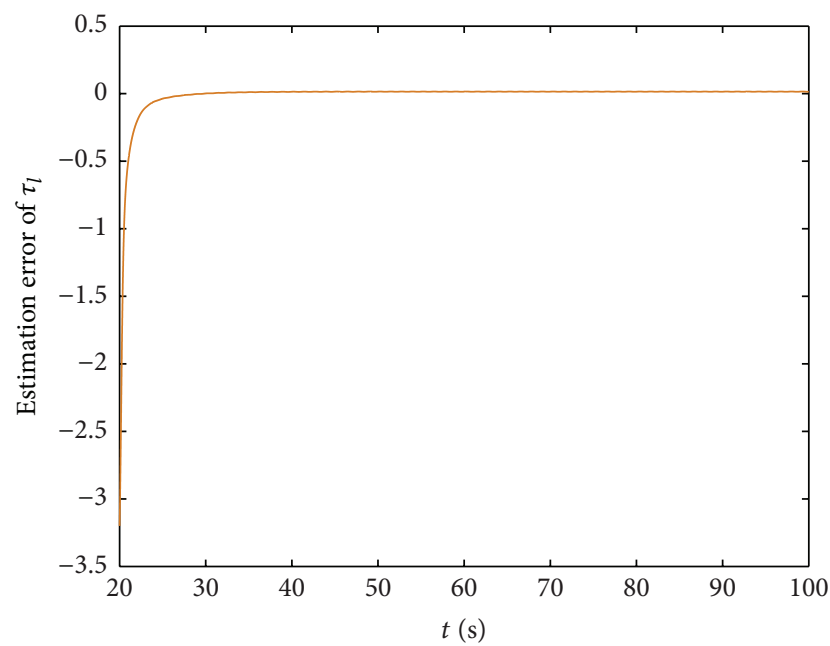

FIGURE 12: The $\widetilde{\tau}_{l}$ curve of estimation error of $\tau_{l}$.

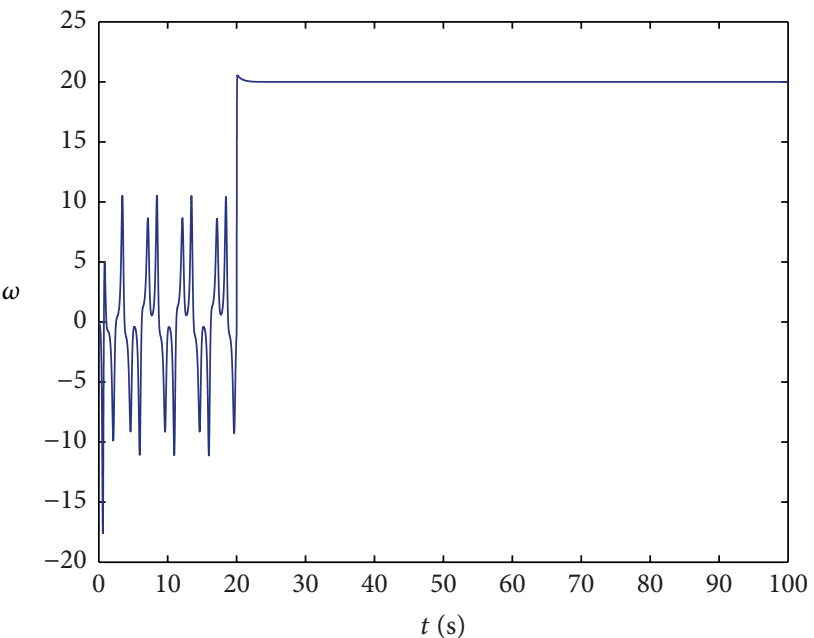

FIGURE 13: The $\omega$ curve of PMSM chaotic system added the controller inputs $u_{d}$ and $u_{q}$.

Figures 5-7 indicate the proposed controller shown in Figures 8-9 can inhibit the external disturbances.

Figures 10-12 show the estimated errors $\tilde{\delta}, \tilde{\gamma}$, and $\tilde{\tau}_{l}$ of unknown parameters $\delta, \gamma$, and $\tau_{l}$ for PMSM chaotic system, which testify the effectiveness of constructed adaptive laws and demonstrate the proposed approach has a good robustness against the uncertainties in system parameters.

4.2. Test-II. In reality, the motor parameters are frequently varying with the design values. As a result, the parameters $\delta, \gamma$, and $\tau_{l}$ in Test-I are changed into $\delta=0.1, \gamma=25$, and $\tau_{l}=1.6$ in Test-II, respectively. Simultaneously, the expected reference signals are also changed and set as $\omega^{*}=20$ and $i_{d}^{*}=0$. In a word, the unknown motor parameters and expected reference signals all differ from Test-I, which is able to validate the proposed control algorithm better. The simulation results are shown in Figures 13-20. Figures 13-15 indicate the motor's output states $\omega, i_{q}$, and $i_{d}$ added the

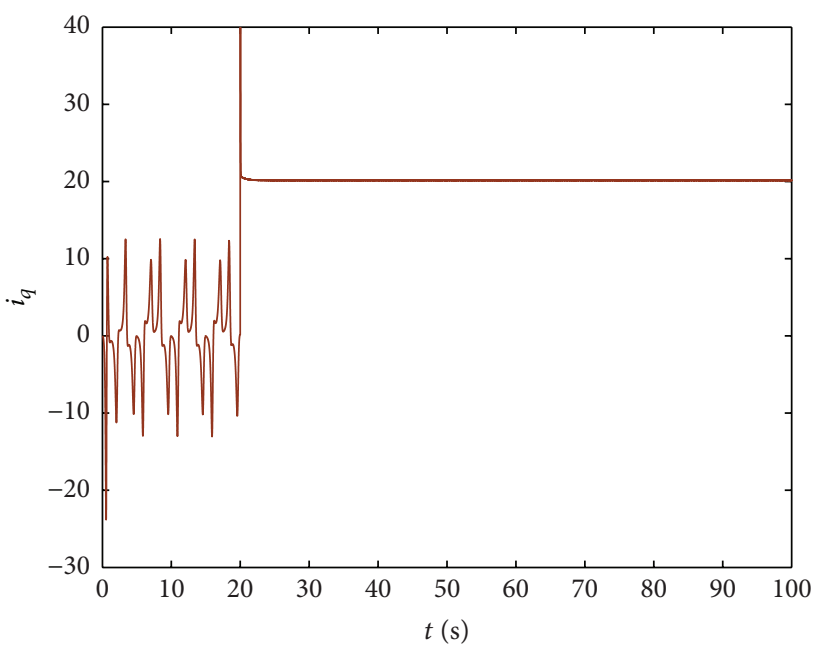

FIGURE 14: The $i_{q}$ curve of PMSM chaotic system added the controller inputs $u_{d}$ and $u_{q}$.

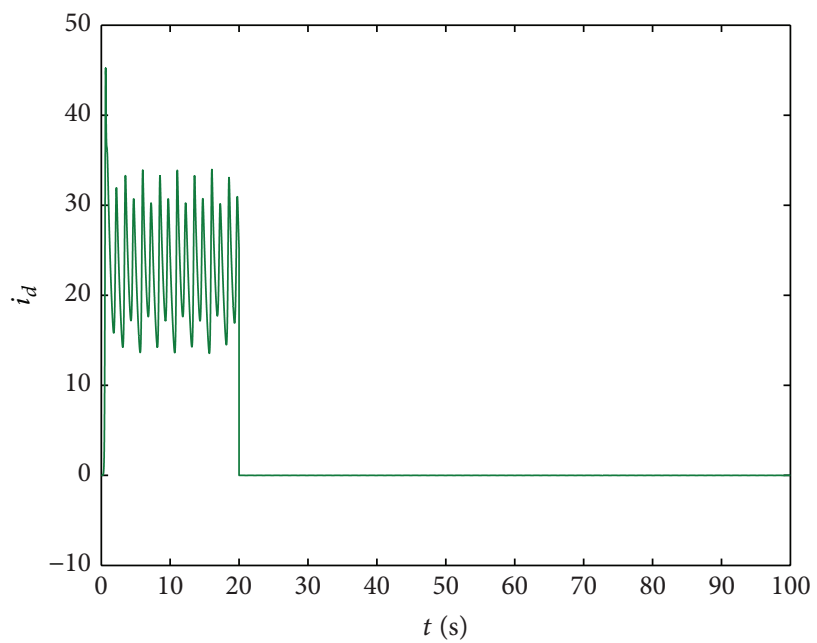

FIgURE 15: The $i_{d}$ curve of PMSM chaotic system added the controller inputs $u_{d}$ and $u_{q}$.

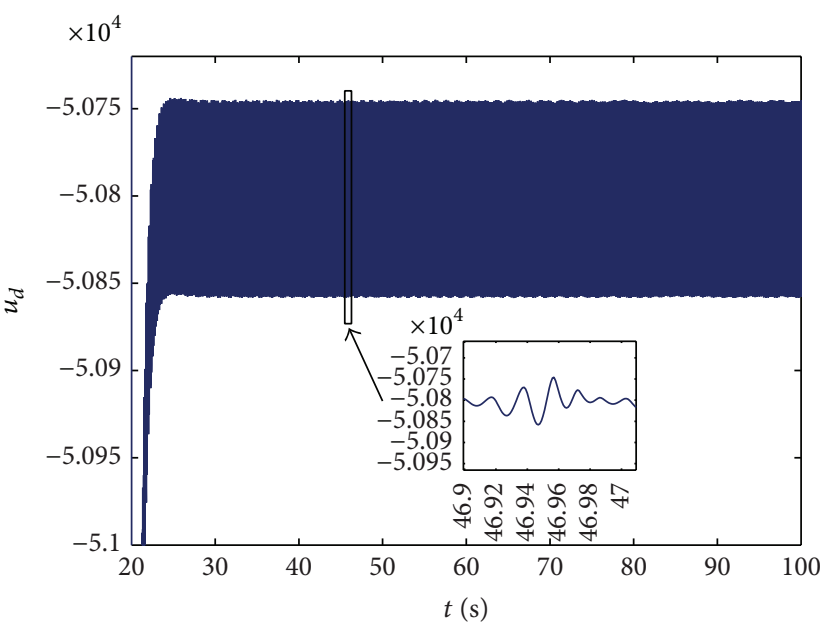

FIGURE 16: The controller input $u_{d}$. 


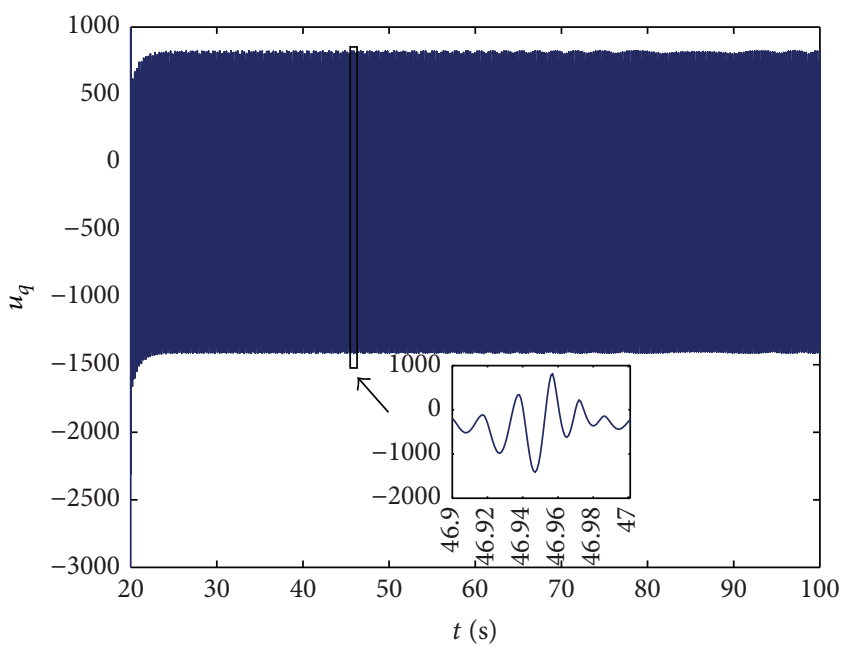

FIGURE 17: The controller input $u_{q}$.

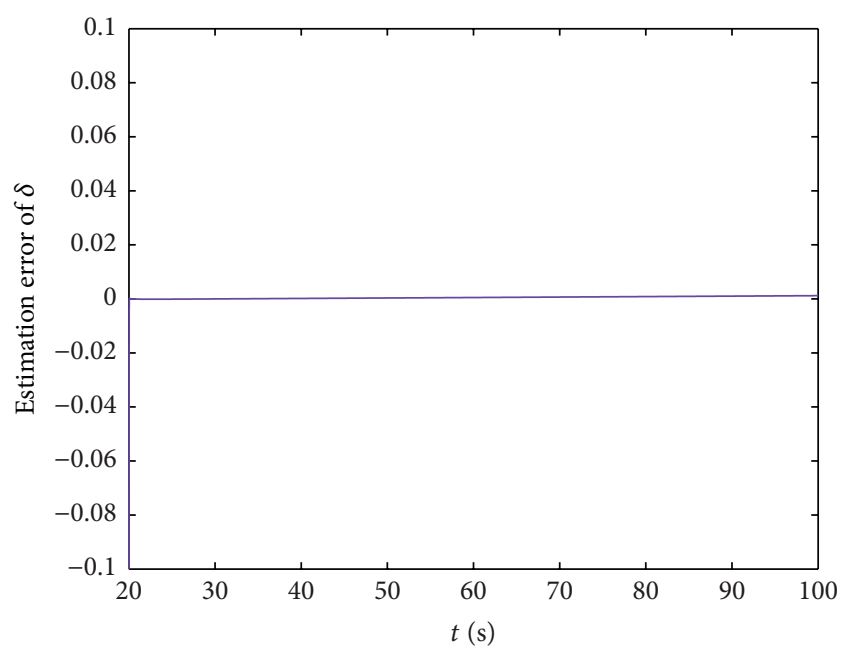

FIgURE 18: The $\widetilde{\delta}$ curve of estimation error of $\delta$.

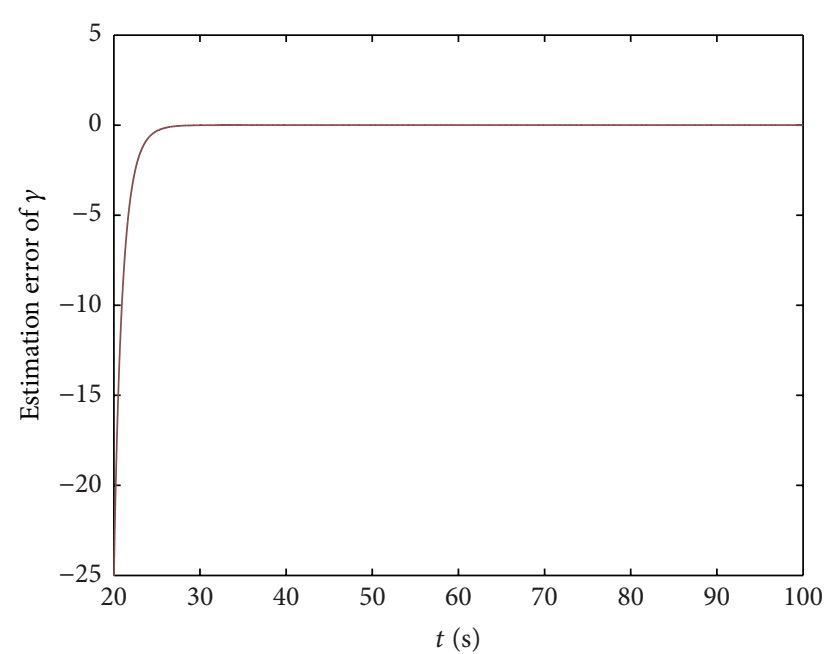

Figure 19: The $\tilde{\gamma}$ curve of estimation error of $\gamma$.

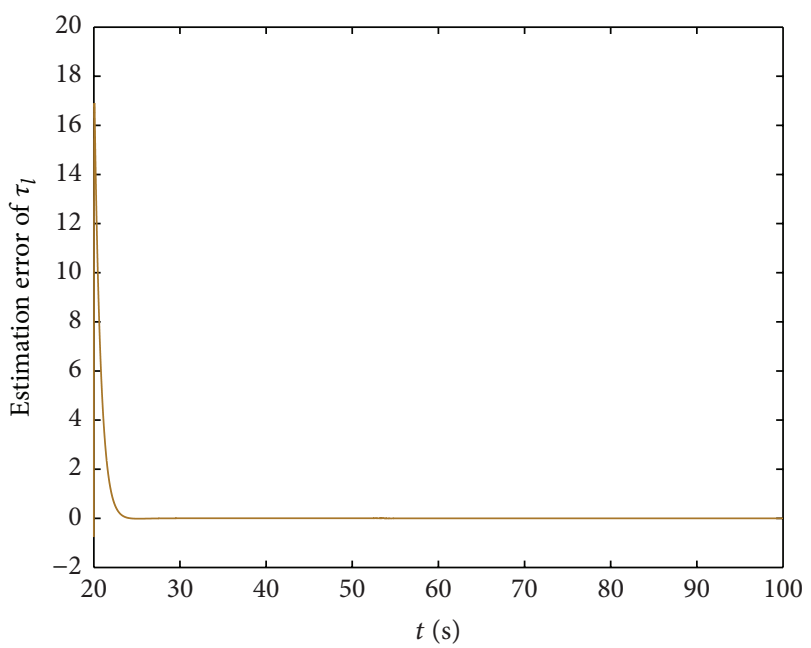

FIGURE 20: The $\widetilde{\tau}_{l}$ curve of estimation error of $\tau_{l}$.

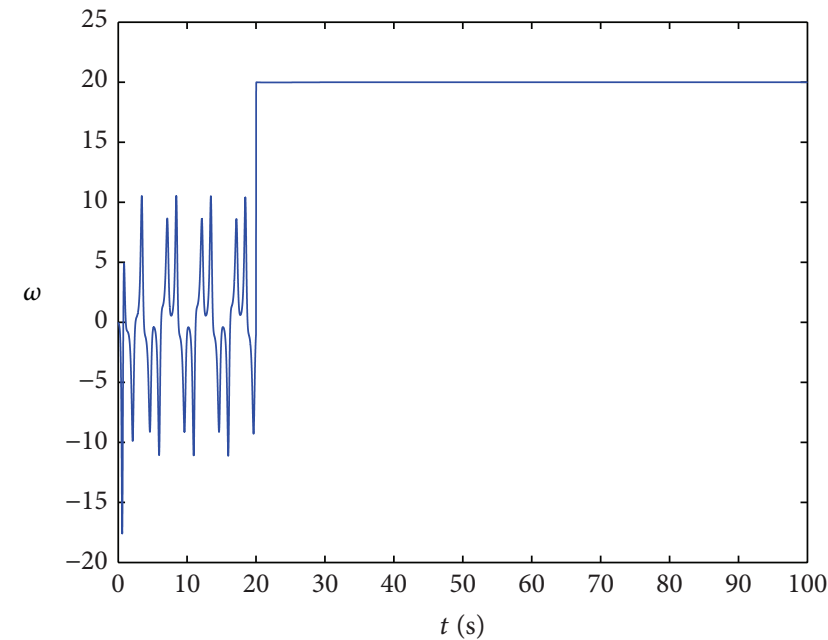

FIGURE 21: The $\omega$ curve of PMSM chaotic system added the controller inputs $u_{d}$ and $u_{q}$.

controller inputs $u_{d}$ and $u_{q}$ shown in Figures 16-17, which demonstrate that the designed controller can guarantee the outputs track references well and suppress the external disturbances effectively. Figures 18-20 indicate that the designed adaptive law can estimate the fully unknown parameters precisely even if the fully unknown parameters are changed.

4.3. Test-III. For Test-III, the external disturbances are enlarged in addition to changing the unknown motor parameters and expected reference signals on the basis of Test-II, which are described as $\Delta_{1}(\mathbf{x}, t)=40 x_{3} \sin (5 t)$ and $\Delta_{2}(\mathbf{x}, t)=$ $20 \sin (5 t)$. The control difficulty in Test-III is larger than the previous two experiments and Test-III is a more general instance to verify the controller's performance. The simulation results are shown in Figures 21-28. Figures 21-23 give the curves of the state variables $\omega, i_{q}$, and $i_{d}$, which manifest these variables are controlled to their references and chaos is eliminated when adding the proposed controllers 


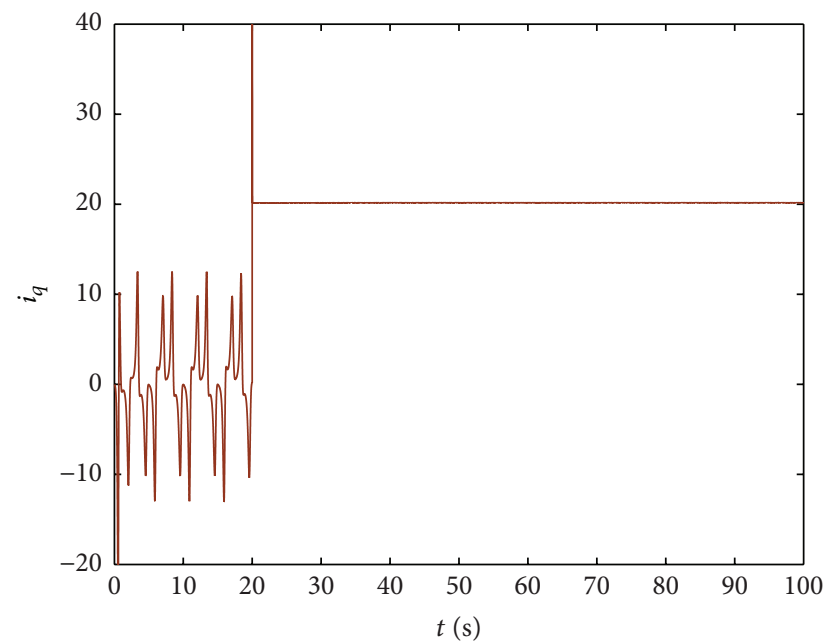

FIGURE 22: The $i_{q}$ curve of PMSM chaotic system added the controller inputs $u_{d}$ and $u_{q}$.

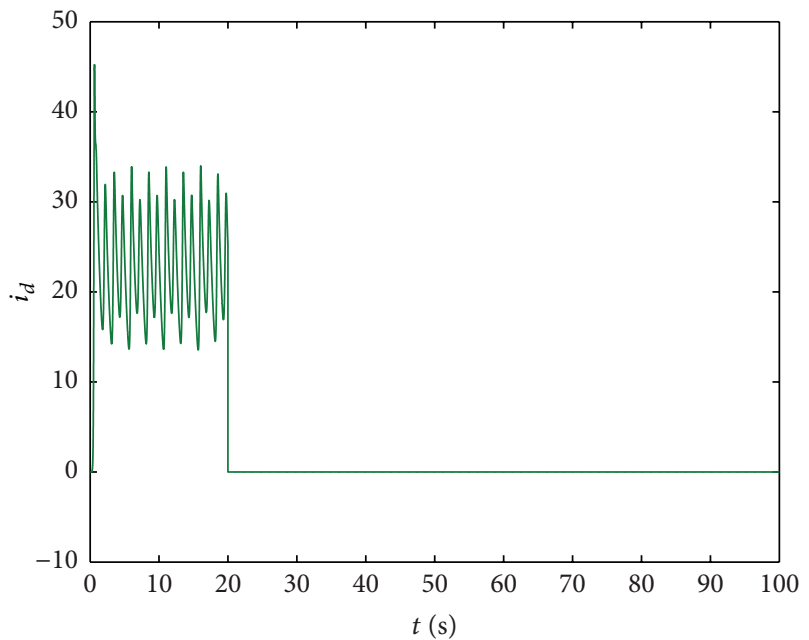

FIGURE 23: The $i_{d}$ curve of PMSM chaotic system added the controller inputs $u_{d}$ and $u_{q}$.

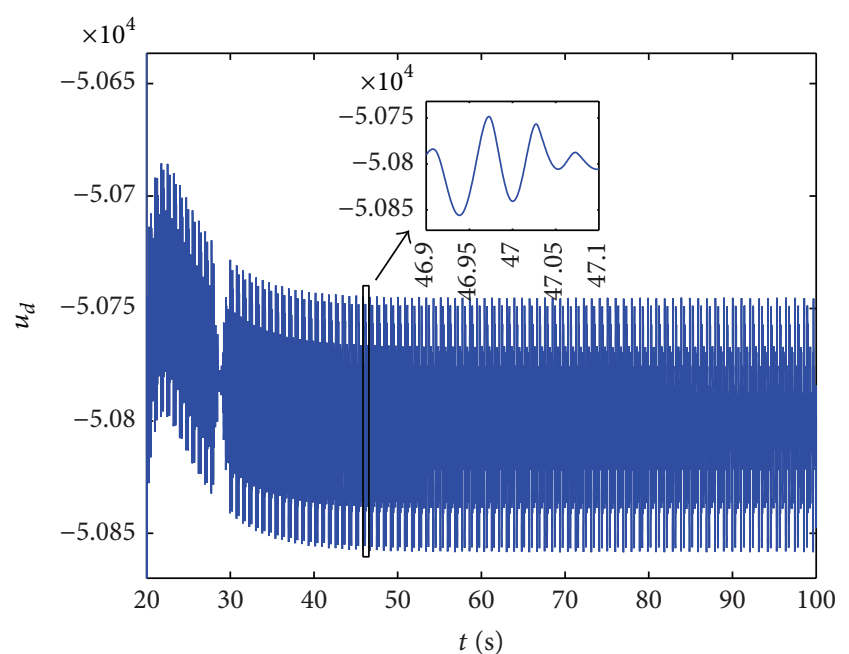

Figure 24: The controller input $u_{d}$.

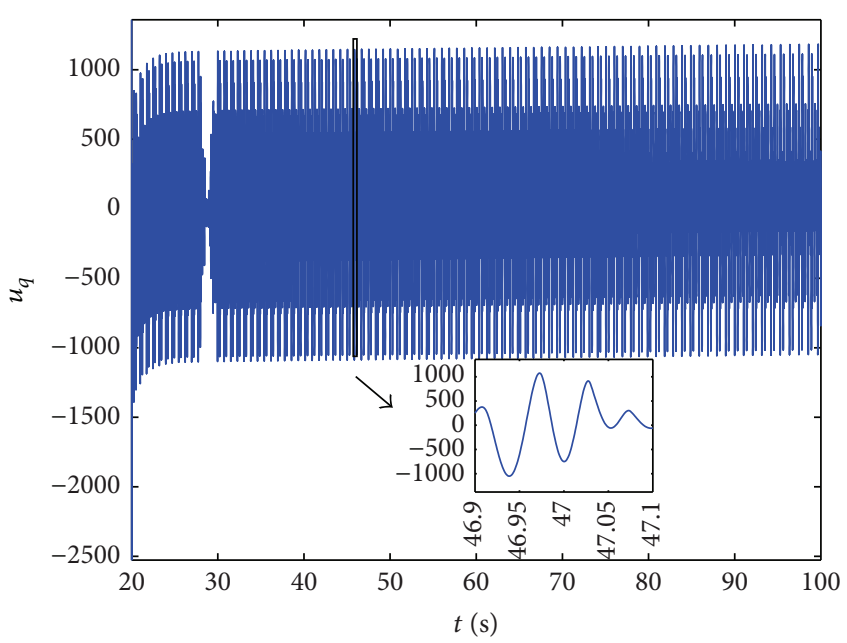

FIGURE 25: The controller input $u_{q}$.

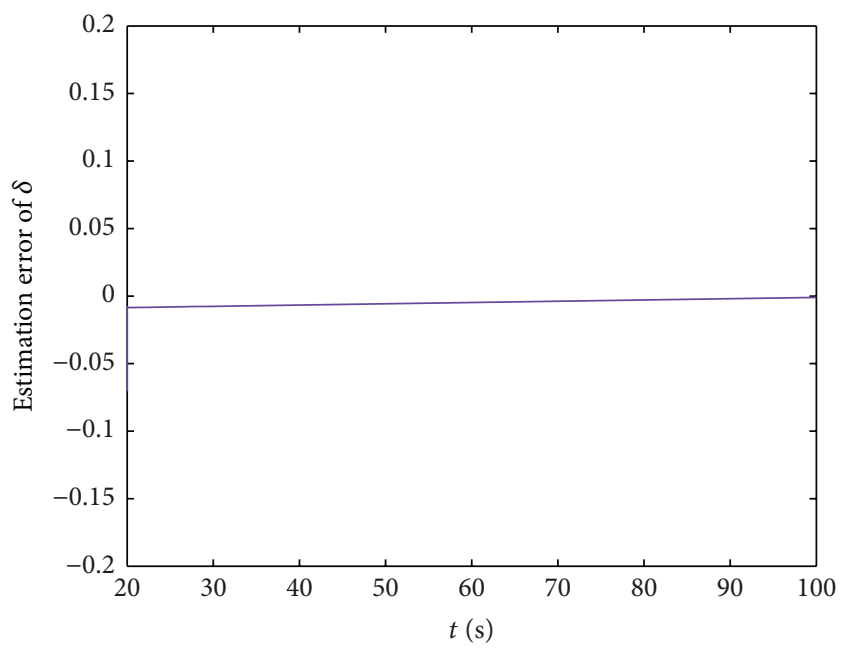

FIGURE 26: The $\widetilde{\delta}$ curve of estimation error of $\delta$.

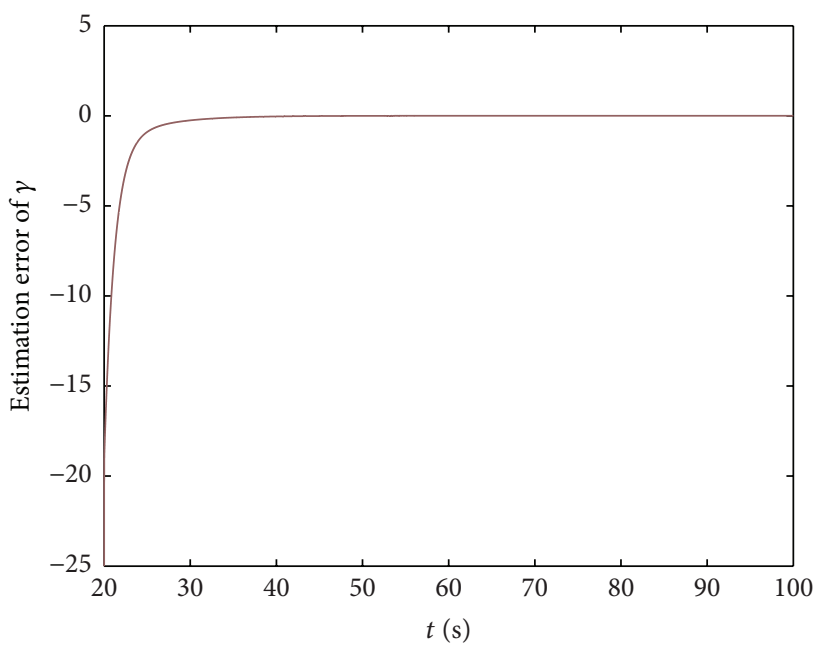

Figure 27: The $\tilde{\gamma}$ curve of estimation error of $\gamma$. 


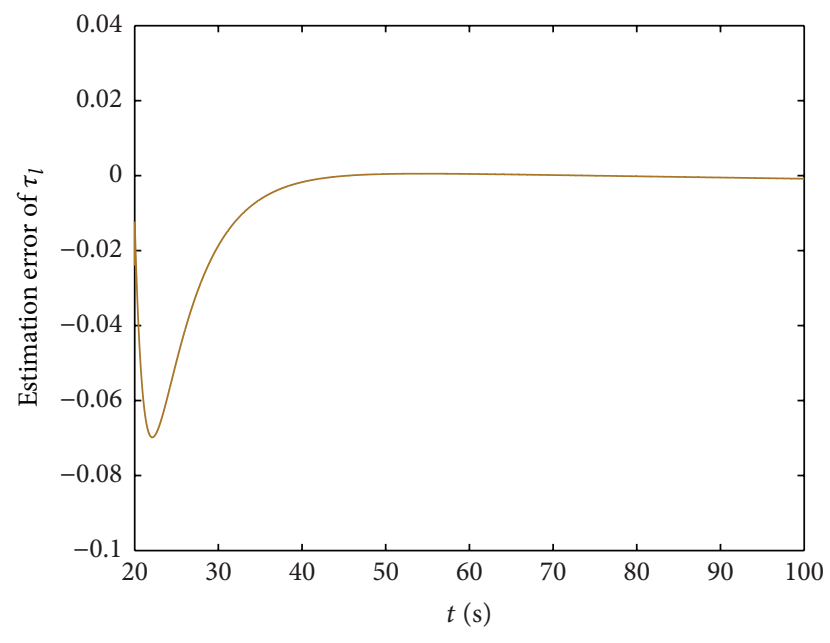

FIGURE 28: The $\widetilde{\tau}_{l}$ curve of estimation error of $\tau_{l}$.

$u_{d}$ and $u_{q}$ shown in Figures 24-25. Figures 21-23 also illustrate the enlarged external disturbances are restrained by the controllers. The estimation errors of the fully unknown parameters are provided in Figures 26-28, which proves the effectiveness of the adaptive laws again.

Remark 5. Previous researches on parameter estimation of PMSM chaotic system mostly assumed that only partial parameters of the system are unknown. The paper takes fully nondeterministic parameters $\delta, \gamma$, and $\tau_{l}$ into account; it undoubtedly extends the theory of parameter estimation for PMSM chaotic system.

Remark 6. The action time of control inputs is $20 \mathrm{~s}$ in the simulation. The aim of doing this is to observe the effect of the control approach better. In reality, as long as the chaos occurs, the controller will be put into effect.

Remark 7. On the basis of considering fully unknown parameters, the external disturbances are introduced into the PMSM chaotic model. Hence, the designed control consists of two parts. One is to guarantee the state variables to track the reference signals; another is to suppress the external disturbances. In general, the simultaneous consideration of fully unknown parameters and external disturbances makes the research results more general and practical.

\section{Conclusions}

In this paper, a control approach is proposed to address the control issue of chaos in PMSM system with fully unknown parameters and external disturbances. Main conclusions are acquired as the following:

(1) Through combination of adaptive control with backstepping control, the presented adaptive robust backstepping control scheme resolves the main problems of the conventional backstepping algorithm encountered. And the stability of the designed controller is proved by Lyapunov theory.
(2) The simulation results show that the designed controller is able to make the PMSM operate out of chaotic state quickly, and the adaptive laws are established to estimate the unknown parameters accurately. Furthermore, the proposed algorithm can ensure the unknown parameters converge to the actual values fast and restrain the external disturbance effectively.

(3) The design method in this paper is simple and effective. For PMSM chaotic system with fully unknown parameters, the control variables in proposed approach can be self-adjusted with the changing of system parameters. Therefore, our findings are more practical and more convenient for engineering applications. Future research will discuss the application of the proposed control approach into practical implementation.

\section{Competing Interests}

The author declares that there is no conflict of interests regarding the publication of this paper.

\section{Acknowledgments}

This work is partially supported by the National Natural Science Foundation of China (Grant no. 51407077) and the Fundamental Research Funds for the Central Universities of Ministry of Education of China (Grant no. 2014MS93).

\section{References}

[1] N. Hemati, "Strange attractors in brushless DC motors," IEEE Transactions on Circuits and Systems I: Fundamental Theory and Applications, vol. 41, no. 1, pp. 40-45, 1994.

[2] Z. Li, J. B. Park, Y. H. Joo, B. Zhang, and G. Chen, "Bifurcations and chaos in a permanent-magnet synchronous motor," IEEE Transactions on Circuits and Systems I: Fundamental Theory and Applications, vol. 49, no. 3, pp. 383-387, 2002.

[3] Z. Jing, C. Yu, and G. Chen, "Complex dynamics in a permanent-magnet synchronous motor model," Chaos, Solitons and Fractals, vol. 22, no. 4, pp. 831-848, 2004.

[4] C.-L. Li, S.-M. Yu, and X.-S. Luo, "Fractional-order permanent magnet synchronous motor and its adaptive chaotic control," Chinese Physics B, vol. 21, no. 10, Article ID 100506, 2012.

[5] Y.-Y.Hou, "Finite-time chaos suppression of permanent magnet synchronous motor systems," Entropy, vol. 16, no. 4, pp. 22342243, 2014.

[6] H. P. Ren and D. Liu, "Nonlinear feedback control of chaos in permanent magnet synchronous motor," IEEE Transactions on Circuits and Systems II: Express Briefs, vol. 53, no. 1, pp. 45-50, 2006.

[7] A. Loría, "Robust linear control of (chaotic) permanent-magnet synchronous motors with uncertainties," IEEE Transactions on Circuits and Systems. I. Regular Papers, vol. 56, no. 9, pp. 21092122, 2009.

[8] M. Messadi, A. Mellit, K. Kemih, and M. Ghanes, "Predictive control of a chaotic permanent magnet synchronous generator in a wind turbine system," Chinese Physics B, vol. 24, no. 1, Article ID 010502, 2015. 
[9] Z. Li, B. Zhang, and Z. Y. Mao, "Entrainment and migration control of permanent-magnet synchronous motor system," Control Theory and Applications, vol. 19, no. 1, pp. 53-56, 2002.

[10] H. C. Cho, K. S. Lee, and M. S. Fadali, "Adaptive control of pmsm systems with chaotic nature using lyapunov stability based feedback linearization," International Journal of Innovative Computing, Information and Control, vol. 5, no. 2, pp. 479488, 2009.

[11] D. Q. Wei, B. Zhang, X. S. Luo, S. Y. Zeng, and D. Y. Qiu, "Effects of couplings on the collective dynamics of permanentmagnet synchronous motors," IEEE Transactions on Circuits and Systems II: Express Briefs, vol. 60, no. 10, pp. 692-696, 2013.

[12] G. Maeng and H. H. Choi, "Adaptive sliding mode control of a chaotic nonsmooth-air-gap permanent magnet synchronous motor with uncertainties," Nonlinear Dynamics, vol. 74, no. 3, pp. 571-580, 2013.

[13] T.-B.-T. Nguyen, T.-L. Liao, and J.-J. Yan, "Adaptive sliding mode control of chaos in permanent magnet synchronous motor via fuzzy neural networks," Mathematical Problems in Engineering, vol. 2014, Article ID 868415, 11 pages, 2014.

[14] J. P. Yu, B. Chen, and H. S. Yu, "Fuzzy-approximation-based adaptive control of the chaotic permanent magnet synchronous motor," Nonlinear Dynamics, vol. 69, no. 3, pp. 1479-1488, 2012.

[15] Y.-F. Yang, M.-Z. Luo, S.-B. Xing, X.-X. Han, and H.-Q. Zhu, "Analysis of chaos in permanent magnet synchronous generator and optimal output feedback $H_{\infty}$ control," Acta Physica Sinica, vol. 64, no. 4, Article ID 040504, 2015.

[16] J.-H. Hao, X.-W. Wang, and H. Zhang, "Chaotic robust control of permanent magnet synchronous motor system under uncertain factors," Acta Physica Sinica, vol. 63, no. 22, Article ID 220203, 2014.

[17] S. H. Luo, "Adaptive fuzzy dynamic surface control for the chaotic permanent magnet synchronous motor using Nussbaum gain," Chaos, vol. 24, no. 3, Article ID 033135, 2014.

[18] D. Q. Wei, X. S. Luo, B. H. Wang, and J. Q. Fang, "Robust adaptive dynamic surface control of chaos in permanent magnet synchronous motor," Physics Letters A, vol. 363, no. 1-2, pp. 7177, 2007.

[19] A. N. Njah, "Tracking control and synchronization of the new hyperchaotic Liu system via backstepping techniques," Nonlinear Dynamics, vol. 61, no. 1-2, pp. 1-9, 2010.

[20] X. Wang and J. Zhang, "Tracking control and the Backstepping design of synchronization controller for Chen system," International Journal of Modern Physics B, vol. 25, no. 28, pp. 3815-3824, 2011.

[21] N. Chen, S. Q. Xiong, B. Liu, and W. H. Gui, "Adaptive backstepping control of permanent magnet synchronous motor chaotic system," Journal of Central South University (Science and Technology), vol. 45, no. 1, pp. 99-104, 2014.

[22] X. Ge and J. Huang, "Chaos control of permanent magnet synchronous motor," in Proceedings of the 8th International Conference on Electrical Machines and Systems, pp. 484-488, Nanjing, China, September 2006.

[23] C. M. Kwan and F. L. Lewis, "Robust backstepping control of induction motors using neural networks," IEEE Transactions on Neural Networks, vol. 11, no. 5, pp. 1178-1187, 2000.

[24] A. Stotsky, I. Kanellakopoulus, and P. Kokotovic, Nonlinear and Adaptive Control Design, John Wiley \& Sons, New York, NY, USA, 1995.

[25] A. Harb and W. Ahmad, "Control of chaotic oscillators using a nonlinear recursive backstepping controller," in Proceedings of the IASTED Conference on Applied Simulations and Modeling, pp. 451-453, Crete, Greece, June 2002.

[26] J.-H. Hu and J.-B. Zou, "Adaptive backstepping control of permanent magnet synchronous motors with parameter uncertainties," Control and Decision, vol. 21, no. 11, pp. 1264-1269, 2006 


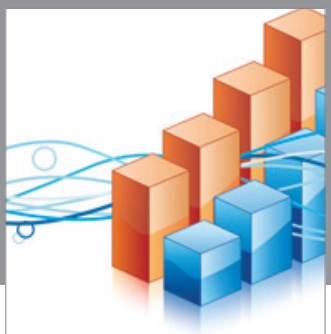

Advances in

Operations Research

vatem alat4

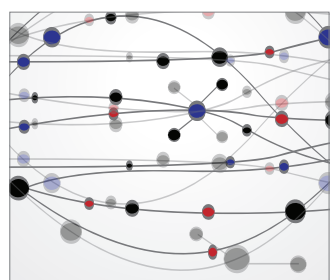

\section{The Scientific} World Journal
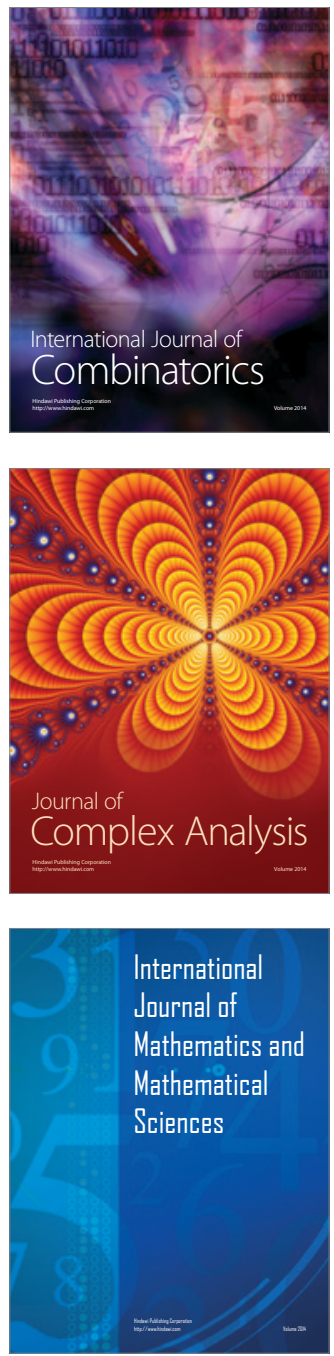
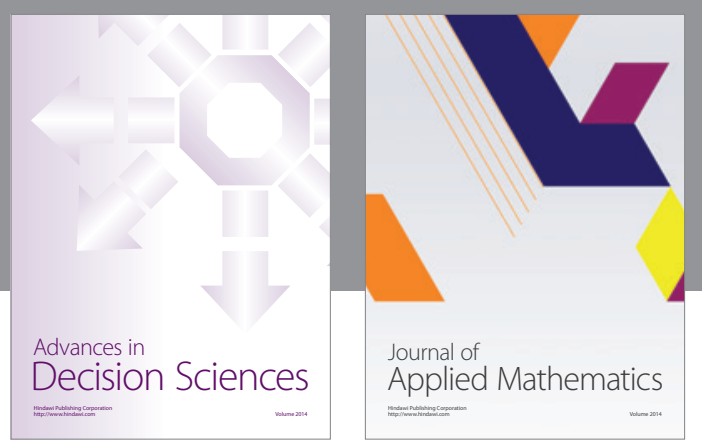

Algebra

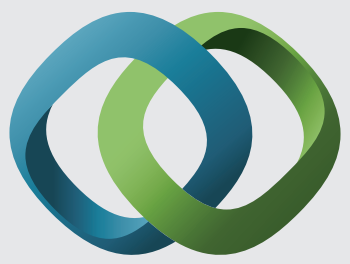

\section{Hindawi}

Submit your manuscripts at

http://www.hindawi.com
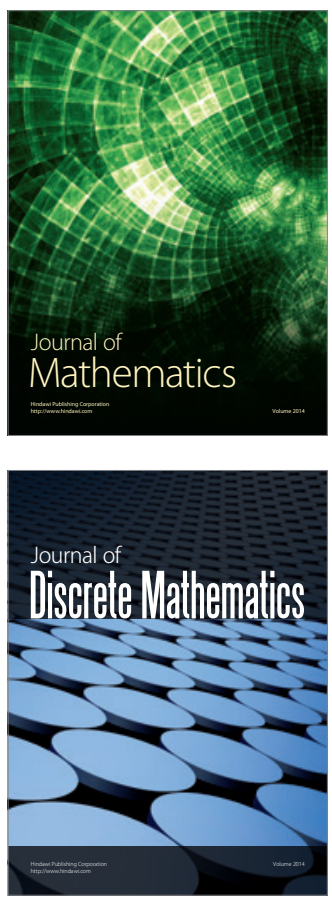

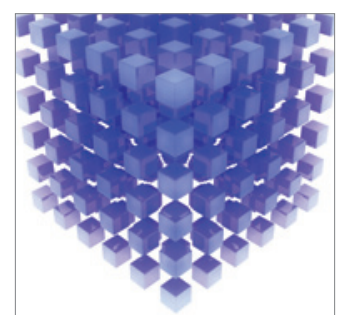

Mathematical Problems in Engineering
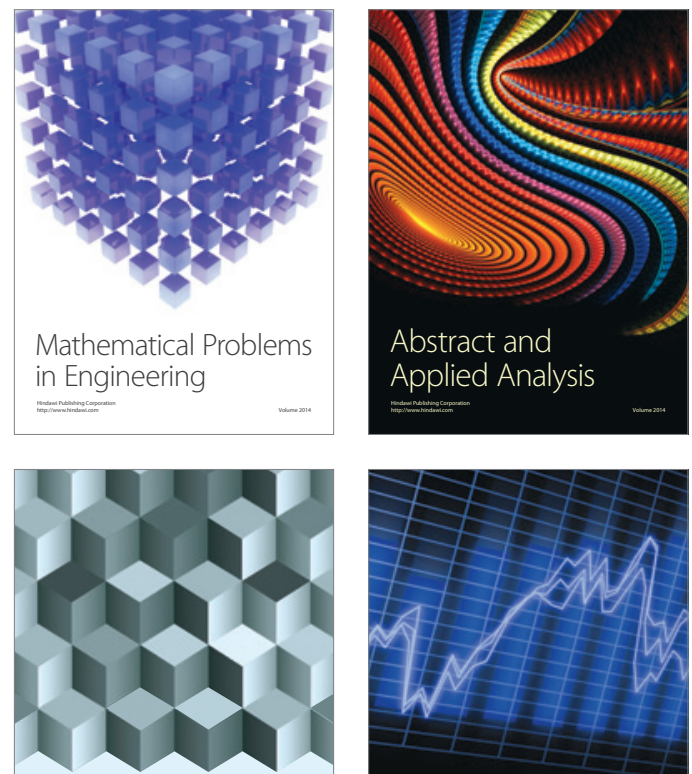

Journal of

Function Spaces

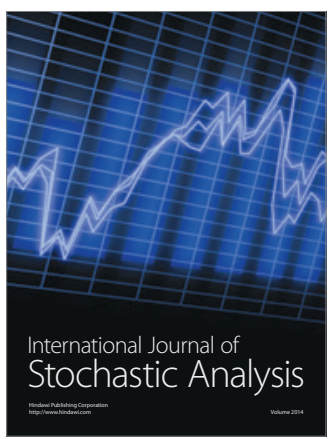

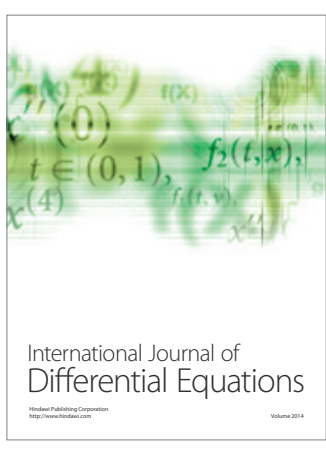
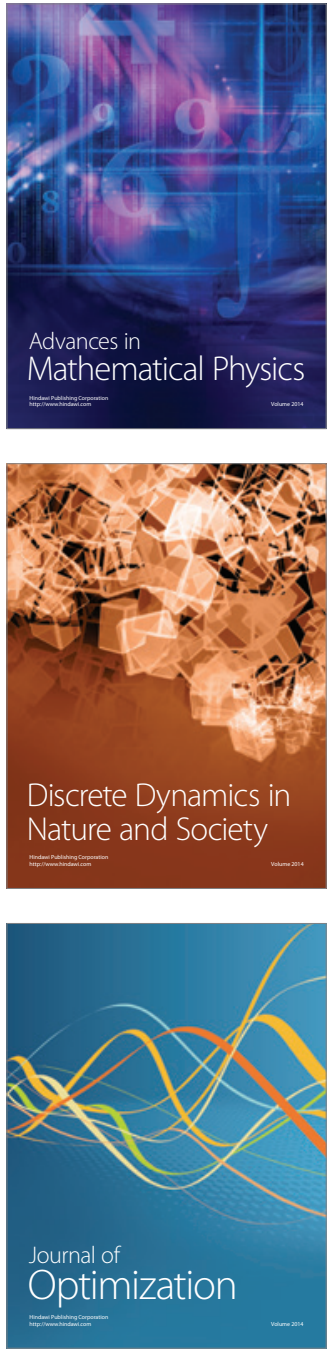